\title{
Analysis of Traffic Evolution on Road Networks of a Roundabout
}

\author{
Tolesa Hundesa Muleta ${ }^{1}$ and Legesse Lemecha Obsu $\mathbb{D}^{2}$ \\ ${ }^{1}$ Department of Mathematics, Hawassa University, Hawassa, Ethiopia \\ ${ }^{2}$ Department of Mathematics, Adama Science and Technology University, Adama, Ethiopia \\ Correspondence should be addressed to Legesse Lemecha Obsu; legesse.lemecha@astu.edu.et
}

Received 30 May 2020; Accepted 11 September 2020; Published 5 October 2020

Academic Editor: Yun-Bo Zhao

Copyright ( $\odot 2020$ Tolesa Hundesa Muleta and Legesse Lemecha Obsu. This is an open access article distributed under the Creative Commons Attribution License, which permits unrestricted use, distribution, and reproduction in any medium, provided the original work is properly cited.

\begin{abstract}
In this paper, the analyses of traffic evolution on the road network of a roundabout having three entrances and three exiting legs are conducted from macroscopic point of view. The road networks of roundabouts are modeled as a merging and diverging types $1 \times 2$ and $2 \times 1$ junctions. To study traffic evolution at junction, two cases have been considered, namely, demand and supply limited cases. In each case, detailed mathematical analysis and numerical tests have been presented. The analysis in the case of demand limited showed that rarefaction wave fills the portion of the road network in time. In the contrary, in supply limited case, traffic congestion occurs at merging junctions and shock wave propagating back results in reducing the performance of a roundabout to control traffic dynamics. Also, we illustrate density and flux profiles versus space discretization at different time steps via numerical simulation with the help of Godunov scheme.
\end{abstract}

\section{Introduction}

Macroscopic traffic flow model was first introduced in the 1950s due to the seminal work of Lighthill and Whitham [1] and independently by Richards [2]. The model describes the progression of traffic density by the means of macroscopic characteristic governed by scalar conservation law [3-9]. Such modeling approach is also known as LWR model in traffic literature and has been extended to road network with incorporating appropriate boundary condition at junction by several authors, see for example in [10-13] and references cited therein.

Worldwide, traffic congestion has been a significant challenging problem related to transportation in most urban areas. This is mainly due to rapid increase of motorizations and limited carrying capacity of urban road networks. Congestion is worsen at road intersections during peak hours. Thus, congestion leads to delays in the movement of both goods and passengers $[14,15]$. As a result, it affects the devel- opment of a national economy [16]. Real-time and precise prediction models are capable of analyzing traffic flow trends and characteristics on urban road network $[17,18]$.

Roundabouts are special road network with a onedirectional circulatory around a central island. It is another means of regulating traffic evolutions at road intersections rendering better safety relative to conventional road intersections [19]. A three entering and three exiting roundabout can be described as a chain of junctions with two incoming and one exiting roads and one incoming and two exiting roads [20]. The dynamics of traffic on the road network of a roundabout is assumed to be governed by nonlinear scalar hyperbolic conservation laws. Usually, vehicles on the main lane of the roundabout have priority over those entering a junction from external entering roads [21].

The aim of this paper is to investigate traffic evolution on the road networks of a roundabout having three entering and three exiting roads by extending the work introduced in [22] Then, we analyze the efficiency of a roundabout in regulating 
traffic dynamics under different priority parameters. We used Godunov scheme [23] for simulation purposed as detailed in [24].

The content of this work is structured as follows. In Section 2, we present a mathematical model describing traffic evolution on a road network of a roundabout having three entering and three exiting roads. In Section 3, we discuss Riemann Solver at junctions. In Section 4, we analyze the traffic evolution on the road networks of a roundabout both in demand and supply limited cases, respectively. The obtained analytical results are illustrated through numerical simulations in Section 5. Finally, we give conclusion in Section 6.

\section{Mathematical Model}

In this section, we describe road networks of a roundabout having three entering and three exiting roads as indicated in Figure 1. As displayed in Figure 1, the road networks of roundabout can be seen as an oriented graph in which roads are represented by arcs and junctions by vertices.

Each road segment forming main lane, entering, and exiting roads of a roundabout can be modeled by intervals $I_{i}=\left[a_{i}, b_{i}\right] \subseteq \mathbb{R}, a_{i}<b_{i}$, and $S_{i}=\left[c_{i}, d_{i}\right] \subseteq \mathbb{R}, c_{i}<d_{i}, i=1, \cdots, 6$ , where $I_{i}$ denotes the finite collection of entering and exiting road segments and $S_{i}$ denotes the collection of roads on the main lane of the roundabout as illustrated in Figure 2(a). In this setting, we assume that $a_{i}$ tends to $-\infty$ for all $i=2 n-1$ and $b_{i}$ tends to $\infty$ for all $i=2 n, n=1,2,3$. For convenience, we also assume that $b_{i}=c_{i}, i=2 n-1$, and $a_{i}=d_{i}, i=2 n, n$ $=1,2,3$. The types of merging and diverging junctions are presented in Figure 2(b).

The dynamics of traffic on each road segment is governed by LWR model given as

$\frac{\partial \rho_{i}}{\partial t}+\frac{\partial f_{i}\left(\rho_{i}\right)}{\partial x}=0,(t, x) \in \mathbb{R}^{+} \times I_{i},(t, x) \in \mathbb{R}^{+} \times S_{i}, \forall i=1,2, \cdots, 6$

where $\rho_{i}=\rho_{i}(t, x) \in\left[0, \rho_{\text {max }, i}\right]$ is the mean traffic density and $\rho_{\text {max }, i}$ is the maximal traffic density allowed on each road. The speed-density relationship is given by

$$
v_{i}(\rho)=v_{\max , \mathrm{i}}\left(1-\frac{\rho_{i}}{\rho_{\max , \mathrm{i}}}\right),
$$

where $v_{\max , \mathrm{i}}$ is the maximal speed on each road and $v_{i}:[0$ , $\left.\rho_{\max , i}\right] \rightarrow \mathbb{R}^{+}$is a smooth decreasing function denoting the mean traffic speed. The flux density relationship is defined by

$f_{i}\left(\rho_{i}\right)=\rho_{i} v\left(\rho_{i}\right)$, or equivalently, $f_{i}\left(\rho_{i}\right)=v_{\max , \mathrm{i}} \rho_{i}\left(1-\frac{\rho_{i}}{\rho_{\max , \mathrm{i}}}\right)$.

The flux is maximum at $\rho_{c, i} \in\left(0, \rho_{\max , i}\right)$. In this paper, we use the normalized form of the vehicle density $\rho(t, x)$ to be $0 \leq \rho \leq 1$, and we assume the following:
(1) $\rho_{\max , i}=1$

(2) The speed $v_{i}$ depends only on the density $\rho_{i}$

(3) The flux $f_{i}$ is a strictly concave $C^{2}$ function

(4) $f_{i}(0)=f_{i}(1)=0$

Assumptions (A3) and (A4) imply that $f_{i}$ has a unique maximum point $\rho_{c, i} \in(0,1)$ for all $i=1,2,3, \cdots 6$. .

We briefly recall the following definition to be used in later sections.

Definition 1 (see [14]). Let $\tau:[0,1] \rightarrow[0,1]$ be the map such that

(i) $f(\tau(\rho))=f(\rho)$ for every $\rho \in[0,1]$ and $\tau(\rho) \neq \rho$ for every $\rho \in[0,1] \backslash\left\{\rho_{c}\right\}$.

Definition 2. Consider a roundabout with 6 road segments on the main lane with 3 entrance and 3 exit links as given in Figure 1(b). A collection of functions $\left(\rho_{i}\right), i=1, \cdots, 12 \epsilon$ $\prod_{i=1}^{12} C^{0}\left(\mathbb{R}^{+} ; L^{1} \cap \mathrm{BV}\left(I_{i}\right)\right)$ is an admissible solution to (1) as in $[20]$ if

(1) $\rho_{i}$ is a weak solution on $I_{i}$, i.e., $\rho_{i}:[0,+\infty) \times I_{i} \rightarrow[0$ , $\left.\rho_{\text {max }, i}\right]$, such that

$$
\int_{\mathbb{R}^{+}} \int_{I_{i}}\left(\rho_{i} \partial_{t} \varphi_{i}+f\left(\rho_{i}\right) \partial_{x} \varphi_{i}\right) d x d t=0,
$$

for every $\varphi_{i} \in C_{c}^{1}\left(\mathbb{R}^{+} \times I_{i}\right), i=1, \cdots, 12$.

(2) $\rho_{i}$ satisfies the Kruzhkov entropy condition [25] on $\left(\mathbb{R} \times I_{i}\right)$, that is

$$
\begin{gathered}
\int_{\mathbb{R}^{+}} \int_{I_{i}}\left(\left|\rho_{i}-k\right| \partial_{t} \varphi_{i}+\operatorname{sgn}\left(\rho_{i}-k\right) \cdot\left(f\left(\rho_{i}\right)-f(k)\right) \partial_{x} \varphi_{i}\right) \mathrm{dxdt} \\
+\int_{I_{i}}\left(\left|\rho_{i, 0}-k\right| \varphi_{i}(0, x) d x \geq 0 .\right.
\end{gathered}
$$

(3) At each junction

$$
\sum_{i=1}^{n} f\left(\rho_{i}(t, b-)\right)=\sum_{j=n+1}^{n+m} f\left(\rho_{j}\left(t, a_{j}+\right)\right),
$$

where $n, m \in\{1,2\}$ and $i$ and $j$ indicate all the links belonging to a junction. In particular, $i$ indicates the incoming links, and $m$ indicates the exiting ones. Also, $b_{i}-$ represents left side of $b_{i}$ while $a_{i}+$ denotes the right side of $a_{i}$. 


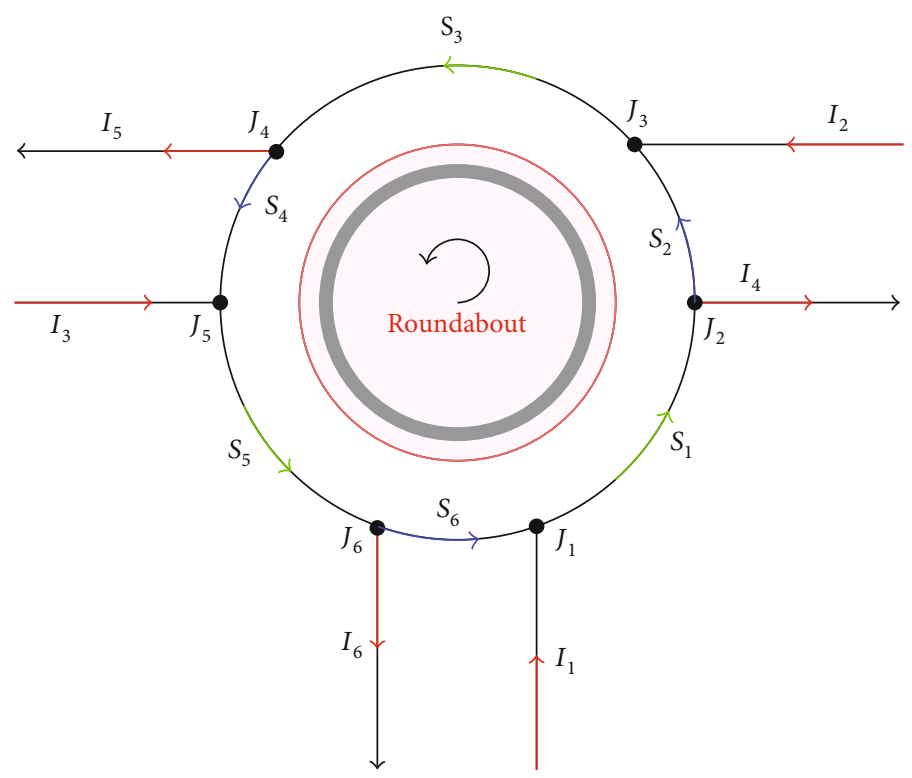

FIGURE 1: Schematic diagram representing a roundabout with 3 entering and 3 exiting roads.

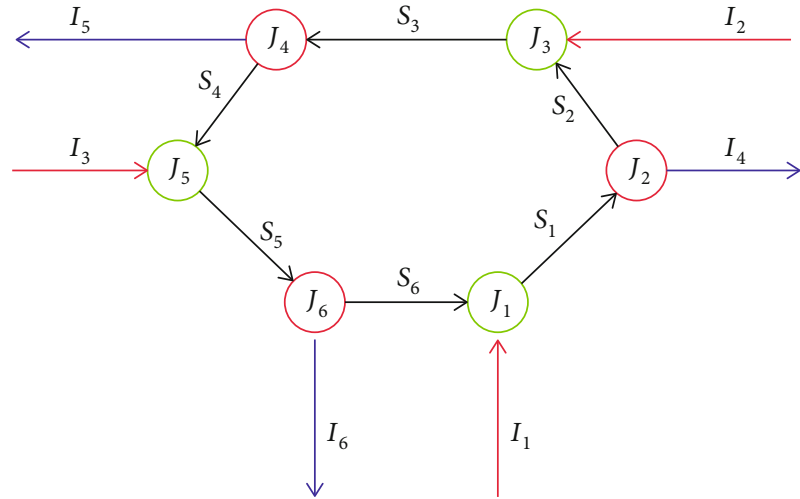

(a) Road network under consideration

\begin{tabular}{cccc}
\hline Junction & Typology & Incoming road & Outgoing road \\
\hline$J_{1}$ & $2 \times 1$ & $I_{1}, S_{6}$ & $S_{1}$ \\
$J_{2}$ & $1 \times 2$ & $S_{1}$ & $I_{4}, S_{2}$ \\
$J_{3}$ & $2 \times 1$ & $I_{2}, S_{2}$ & $S_{3}$ \\
$J_{4}$ & $1 \times 2$ & $S_{3}$ & $I_{5}, S_{4}$ \\
$J_{5}$ & $2 \times 1$ & $I_{3}, S_{4}$ & $S_{5}$ \\
$J_{6}$ & $1 \times 2$ & $S_{5}$ & $I_{6}, S_{6}$ \\
\hline
\end{tabular}

(b) Types of entering and exiting roads at each junction

FIGURE 2: Road networks of a roundabout and its corresponding junction.

\section{Riemann Problem at Junction}

In this section, we briefly describe construction of Riemann solver at junctions.

Definition 3. A Riemann problem at a junction $J$ is a Cauchy problem for constant initial data on each road. Consistency condition:

$$
\operatorname{RS}\left(\operatorname{RS}\left(\rho_{0}\right)\right)=\operatorname{RS}\left(\rho_{0}\right) .
$$

The detail analysis can be found in [11].

Definition 4 (see [20]. A Riemann solver for junction $J$ is a map $R S:[0,1]^{2} \times[0,1] \rightarrow[0,1]^{2} \times[0,1]$ that associates the Riemann data $\rho_{0}=\left(\rho_{1,0}, \rho_{2,0}, \rho_{3,0}\right)$ at $J$ to a vector $\widehat{\rho}=\left(\widehat{\rho}_{1}\right.$, $\left.\widehat{\rho}_{2}, \widehat{\rho}_{3}\right)$ such that the solution on the incoming road $I_{i}, i=1$ , 2 , is given by the wave $\left(\rho_{i}, \widehat{\rho}_{i}\right)$ and on the exiting road $I_{j}, j$ $=3$; the solution is given by the wave $\left(\widehat{\rho}_{j}, \rho_{j}\right)$.

For the entering road, the solution $\rho_{i}(t, x)$ over its particular domain $x<b_{i}$ is given by the solution to the Riemann problem

$$
\left\{\begin{array}{l}
\partial_{t} \rho_{i}+\partial_{x} f\left(\rho_{i}\right)=0 \\
\rho_{i}(0, x)= \begin{cases}\rho_{i, 0} & \text { if } x<b_{i}, \\
\widehat{\rho}_{i} & \text { if } x \geq b_{i} .\end{cases}
\end{array}\right.
$$

Similarly, for the exiting road, the solution $\rho_{j}(t, x)$ over its domain $x>a_{j}$ is given by the solution to the Riemann problem 


$$
\begin{aligned}
& \left\{\begin{array}{l}
\partial_{t} \rho_{j}+\partial_{x} f\left(\rho_{j}\right)=0 \quad(t, x) \in \mathbb{R}^{+} \times I_{j}, \\
\rho_{j}(0, x)= \begin{cases}\rho_{j, 0} & \text { if } x>a_{j}, \\
\widehat{\rho}_{j} & \text { if } x \leq a_{j} .\end{cases} \\
\rho_{1}(0, .) \equiv \rho_{1,0}, \rho_{2}(0, .) \equiv \rho_{2,0}, \rho_{3}(0, .) \equiv \rho_{3,0} .
\end{array}\right.
\end{aligned}
$$

Moreover, there exists a unique $3-\operatorname{tuple}\left(\widehat{\rho}_{1}, \widehat{\rho}_{2}, \widehat{\rho}_{3}\right) \epsilon$ $[0,1]^{3}$ such that

$$
\widehat{\rho}_{i} \in \begin{cases}\left\{\rho_{i, 0}\right\} \cup\left(\tau\left(\rho_{i, 0}\right), 1\right], & \text { if } 0 \leq \rho_{i, 0} \leq \rho_{c}, \\ {\left[\rho_{c}, 1\right],} & \text { if } \rho_{c} \leq \rho_{i, 0} \leq 1 .\end{cases}
$$

On the entering road for $i=1,2$, the solution is given by the wave $\left(\rho_{i, 0}, \widehat{\rho}_{i}\right)$ and

$$
\widehat{\rho}_{j} \in \begin{cases}{\left[0, \rho_{c}\right],} & \text { if } 0 \leq \rho_{j, 0} \leq \rho_{c}, \\ \left\{\rho_{j, 0}\right\} \cup\left[0, \tau\left(\rho_{i, 0}\right)\right), & \text { if } \rho_{c} \leq \rho_{i, 0} \leq 1 .\end{cases}
$$

On the exiting road for $j=3$, the solution is given by the wave $\left(\widehat{\rho}_{3}, \rho_{3,0}\right)$.

On the incoming road $i=1,2$ :

(i) If $\rho_{i, 0}<\rho_{c}<\widehat{\rho}_{i}<1, f\left(\rho_{i, 0}\right)>f\left(\widehat{\rho}_{i}\right)$ and $\rho_{c}<\rho_{i, 0}<1$, then the solution of the Riemann problem consists of a shock wave with a negative speed

(ii) If $\rho_{i, 0}<\rho_{c}<\widehat{\rho}_{i}<1, f\left(\rho_{i, 0}\right)=f\left(\widehat{\rho}_{i}\right)$, then the solution consists of contact wave

$$
\begin{gathered}
\rho_{i, 0} \leq \rho_{c} \Rightarrow \Omega_{i}=\left[0, \gamma_{i, 0}\right], \gamma_{i, 0}=f\left(\rho_{i, 0}\right), \\
\rho_{i, 0} \geq \rho_{c} \Rightarrow \Omega_{i}=\left[0, f\left(\rho_{c}\right)\right] .
\end{gathered}
$$

On the exiting road $j=3$ :

(iii) If $\rho_{j, 0}<\rho_{c}$, then the solution of the Riemann problem consists of a shock wave with a positive speed. If $\widehat{\rho}_{j}<\rho_{c}<\rho_{j, 0}<1$, then the solution of the Riemann problem consists of a shock wave with a positive speed and contact wave when $f\left(\rho_{j, 0}\right)=f\left(\widehat{\rho}_{j}\right)$.

$$
\begin{gathered}
\rho_{j, 0} \leq \rho_{c} \Rightarrow \Omega_{j}=\left[0, f\left(\rho_{c}\right)\right], \quad \gamma_{j, 0}=f\left(\rho_{j, 0}\right), \\
\rho_{j, 0} \geq \rho_{c} \Rightarrow \Omega_{j}=\left[0, \gamma_{j, 0}\right] .
\end{gathered}
$$

3.1. Conditions for Junction Type $1 \times 2$. In this case, we apply traffic distribution ratio $\alpha \in(0,1)$ which express the distribution of traffic among exiting roads based on the preference of drivers at each junction $J$. The Riemann initial data is also denoted by $\rho_{i, 0}=\rho_{i, 0}\left(b_{i}\right)$ for entering roads and $\rho_{j, 0}=\rho_{j, 0}\left(a_{j}\right.$ ) for exiting roads. We represent the unique solution at junc- tion, i.e., at $x=b_{i}$ for entering and $x=a_{i}$ for exiting roads, by $\widehat{\rho}_{1}, \widehat{\rho}_{2}$, and $\widehat{\rho}_{3}$. Traffic flux on the entering road segment is defined by $f\left(\rho_{i, 0}\right)=\gamma_{i, 0}$ satisfying the following Coclite, Garavello, and Piccoli (CGP) conditions $[11,20]$ at junction for each distribution ratio $\alpha_{2,1}, \alpha_{3,1} \in(0,1)$ with $\alpha_{2,1}+\alpha_{3,1}=1$ and convex set $\Omega_{i}$ :

(1) $\gamma_{1} \in \Omega_{1}, \alpha_{j, 1} \gamma_{1} \in \Omega_{j}$ if $j=2,3$.

(2) Maximize $\gamma_{1}$ with respect to (1)

(3) $\gamma_{j}=\alpha_{j, 1} \gamma_{1}, j=2,3$

Using $\Omega_{i}=\left[0, c_{i}\right], i=1,2,3$, we obtain

$$
\gamma_{1}=\min \left\{c_{1}, \frac{c_{2}}{\alpha_{2,1}}, \frac{c_{3}}{\alpha_{3,1}}\right\}
$$

3.2. Conditions for Junction Type $2 \times 1$. In this case, traffic distribution matrix and conservation law alone cannot give unique solution at junction. For this reason, we introduce the priority parameters $p \in(0,1)$ at junction type, $2 \times 1$, two entering and one exiting roads. We assume that all cars cannot enter the exiting road at the time. The purpose of priority parameters is to regulate the condition that neither impose insufficient flows nor send excess vehicles than the carrying capacity of the roads. Assume that $N$ is the number vehicles that can enter the junction. Then, $p N$ cars come from the first entering road and $(1-p) N$ cars from the second entering roads. Then, the solution of the Riemann problem $\left(\rho_{1,0}\right.$, $\left.\rho_{2,0}, \rho_{3,0}\right)$ is formed by a single wave on each road connecting the initial states to $\left(\rho_{1}, \rho_{2}, \rho_{3}\right)$ be determined in the following way. The aim is to maximize traffic flux satisfying:

$$
\widehat{\gamma}_{3}=\min \left\{\gamma_{1}^{\max }\left(\rho_{1,0}\right)+\gamma_{2}^{\max }\left(\rho_{2,0}\right), \gamma_{3}^{\max }\left(\rho_{3,0}\right)\right\} .
$$

Now let $\gamma_{1}=p N$ and $\gamma_{2}=(1-p) N$ be traffic flux on the exiting road such that

$$
\frac{\gamma_{1}}{\gamma_{2}}=\frac{p}{1-p}
$$

Then, it follows that the intersection point of line (16) and $\gamma_{1}+\gamma_{2}=\widehat{\gamma}_{3}$ is occurred at $P$ such that

$$
\Omega=\left\{\left(\gamma_{1}, \gamma_{2}\right): 0 \leq \gamma_{i} \leq \gamma_{i}^{\max }\left(\rho_{i, 0}\right) \leq \gamma_{1}+\gamma_{2} \leq \widehat{\gamma}_{3}\right\}
$$

In the case of Figure 3(a), there is no intersection neither inside nor outside the feasible region. This is occurred in the demand limited case. On the contrary, Figure 3(b) shows intersection occurred inside the feasible region $\Omega$ which indicates supply limited. In the case of Figures 3(c) and $3(\mathrm{~d})$, the intersection point is occurred outside $\Omega$ which indicates supply limited case. A similar analysis study on Riemann solver also discussed in [20].

Note that in Figure 3, $Q$ is the point of segment $\Omega \cap$ $\left\{\left(\gamma_{1}, \gamma_{2}\right): \gamma_{1}+\gamma_{2}=\gamma_{3}{ }_{3}\right\}$ closest to line in equation (16). We compute fluxes at the junction based on the following cases: 


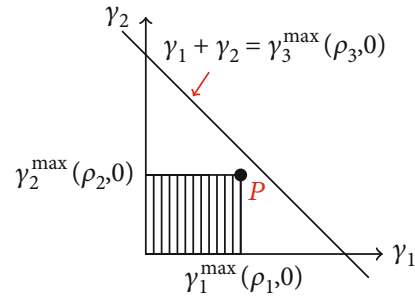

(a) No intersection

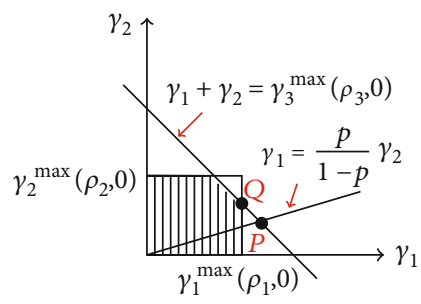

(c) Intersection outside and lowerside

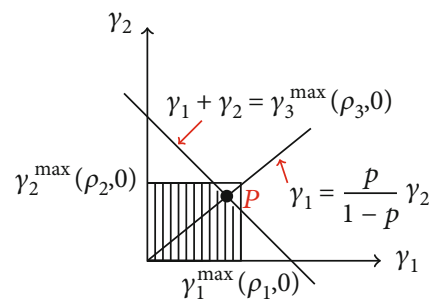

(b) Intersection inside

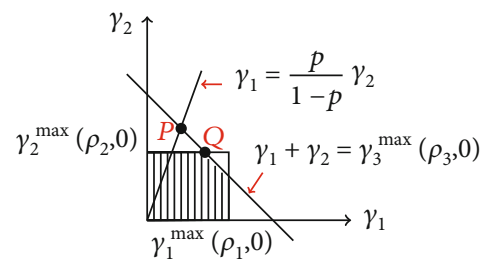

(d) Intersection outside and upperside

FIGURE 3: Cases that illustrates feasible set for solution of the Riemann solver at junctions.

(1) If $c_{1}+c_{2} \leq c_{3}$, we have to look for $\gamma_{1}$ and $\gamma_{2}$ such that

$$
\begin{gathered}
\max \gamma_{1}+\gamma_{2}, \\
\text { w.r.t : } 0 \leq \gamma_{1} \leq c_{1}, 0 \leq \gamma_{2} \leq c_{2}, \gamma_{1}+\gamma_{2} \leq c_{3} .
\end{gathered}
$$

The unique solution is found to be $\gamma_{1}=c_{1}, \gamma_{2}=c_{2}$, and $\gamma_{3}=c_{1}+c_{2}$.

(2) If $c_{1}+c_{2} \geq c_{3}$, we have to look for $\gamma_{1}$ and $\gamma_{2}$ such that

$$
\begin{gathered}
\max \quad \gamma_{1}+\gamma_{2}, \\
\text { w.r.t : } \quad \gamma_{1}=\frac{p}{1-p} \gamma_{2}, 0 \leq \gamma_{1} \leq c_{1}, 0 \leq \gamma_{2} \leq c_{2}, \gamma_{1}+\gamma_{2} \leq c_{3} .
\end{gathered}
$$

\section{Roundabout with Demand and Supply Limited}

To study the nature of traffic evolution on the roundabout, we focus separately in the case of light traffic and heavy traffic.

Assumption 5. To avoid unnecessary complexity, we make the following assumptions.

(1) Complete turn or U-turn on the roundabout is not allowed

(2) There is no obstacle at the exit road of the roundabout

(3) Initially, the network of the roundabout is empty

The model proposed in [22] is applied to a traffic circle with two entering and two exiting roads. The authors computed asymptotic fluxes and then compared the result with traffic light. Analyzing traffic dynamics in the case of traffic circle with two entering and two exiting is very simple compared to a roundabout with three entering and three exiting roads. Further, the priority parameter is applied only at two junctions which is easily computed. In the case of a roundabout with three entering and three exiting roads, computing shock wave on the roundabout is very complicated. So, in this section, we will analyze shock wave on the roundabout via demand and supply limited cases.

4.1. Demand Limited Case. In this case, we assume that traffic arriving at junctions of a roundabout as illustrated in Figure 1 is less, in the sense that the number of cars reaching the roundabout is less than the optimal carrying capacity of a roundabout. Thus, the junction stays demand limited, and we do not need priority rule in this situation. The traffic evolution is only governed by conservation law. However, we assign traffic distribution matrix to describe how traffic coming from the entering roads $S_{i}, i=2 n-1, n=1,2,3$, choose to distribute to their corresponding intermediate roads and external exiting roads. Hence, we have the following assumptions [11].

Assumption 6. We assume three fixed parameter $\alpha_{i} \in(0,1)$ for $i=1,2,3$ such that

(a) If $N$ cars reach the roundabout from $I_{1}$, then $\alpha_{1} N$ drive to road $I_{4}$ and $\left(1-\alpha_{1}\right) N$ drive to road $I_{5}$

(b) If $N$ cars reach the roundabout from $I_{2}$, then $\alpha_{1} N$ drive to road $I_{5}$ and $\left(1-\alpha_{1}\right) N$ drive to road $I_{6}$

(c) If $N$ cars reach the roundabout from $I_{3}$, then $\alpha_{1} N$ drive to road $I_{6}$ and $\left(1-\alpha_{1}\right) N$ drive to road $I_{4}$

Let $\bar{\rho}_{1}, \bar{\rho}_{2}$, and $\bar{\rho}_{3}$ be constant densities on the external entering roads $I_{i}, i=1,2,3$, respectively. In the demand limited case, this situation is equivalent to equilibrium state. In this setting, we define periodic boundary condition at junction as 


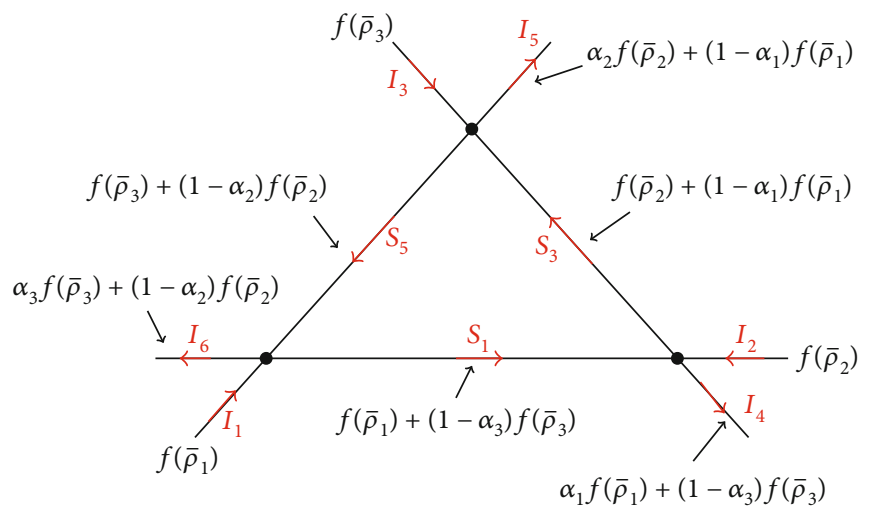

Figure 4: Free-body diagram sketch for traffic at equilibrium situation.

$$
\bar{\rho}_{i}\left(t, a_{i}\right) \equiv \bar{\rho}_{i} i=1,2,3 .
$$

Further, let $f$ denotes traffic flux on the roads network of a roundabout. Then, the constant fluxes on each road do not exceed the maximum flux $f\left(\rho_{c}\right)$, that is, $f\left(\bar{\rho}_{1}\right)+\left(1-\alpha_{3}\right) f($ $\left.\bar{\rho}_{3}\right)<f\left(\rho_{c}\right), f\left(\bar{\rho}_{2}\right)+\left(1-\alpha_{1}\right) f\left(\bar{\rho}_{1}\right)<f\left(\rho_{c}\right)$ and $f\left(\bar{\rho}_{3}\right)+(1-$ $\left.\alpha_{2}\right) f\left(\bar{\rho}_{2}\right)<f\left(\rho_{c}\right)$. If $\alpha_{i} f\left(\bar{\rho}_{i}\right)$ of the flux leaves the roundabout at junction $J_{i}$, the remaining $\left(1-\alpha_{i}\right) f\left(\bar{\rho}_{i}\right)$ will continue on the links of a roundabout until reaching the next exiting road as depicted in Figure 4.

Theorem 7. Consider a roundabout with three entering and three exiting roads as displayed in Figure 4. Then, there exists time dependent coefficient $\alpha_{i}:[0, \infty] \rightarrow[0,1), i=1,2,3$. Provided that the boundary condition hold at $t=0$ and the solution $\rho(t)$ is constantly equal to that of Figure 4 for $t \geq T>0$.

Proof. Since by its nature, the traffic behavior is time dependent, and thus, it is common to assume that $\alpha_{i}$ varies with time. This means, we move forward in time to construct the solution of Riemann problem at each junction and joined them together. By assumption, the traffic evolution is light on the roundabout. This implies that the cars from roads $I_{1}, I_{2}$, and $I_{3}$ reach either of the existing roads $I_{4}, I_{5}$, and $I_{6}$ through rarefaction wave. At each time step, rarefaction waves reach either of the junction $J_{1}, J_{3}$, or $J_{5}$. We regulate the corresponding traffic distribution coefficient in such a way that cars reach the correct road. Further, the existence of such coefficient is also depends on driver's preference. Thus, the crossing coefficients at equilibrium situations are

(i) $\left(S_{1}, I_{4}, S_{2}\right)$

$\alpha_{S_{1}, 4}=\frac{\alpha_{1} f\left(\bar{\rho}_{1}\right)+\left(1-\alpha_{3}\right) f\left(\bar{\rho}_{3}\right)}{f\left(\bar{\rho}_{1}\right)+\left(1-\alpha_{3}\right) f\left(\bar{\rho}_{3}\right)}, \alpha_{S_{1}, S_{2}}=\frac{\left(1-\alpha_{1}\right) f\left(\bar{\rho}_{1}\right)}{f\left(\bar{\rho}_{1}\right)+\left(1-\alpha_{3}\right) f\left(\bar{\rho}_{3}\right)}$,

(ii) $\left(S_{3}, I_{5}, S_{4}\right)$

$$
\alpha_{S_{3}, 5}=\frac{\alpha_{2} f\left(\bar{\rho}_{2}\right)+\left(1-\alpha_{1}\right) f\left(\bar{\rho}_{1}\right)}{f\left(\bar{\rho}_{2}\right)+\left(1-\alpha_{1}\right) f\left(\bar{\rho}_{1}\right)}, \alpha_{S_{3}, S_{4}}=\frac{\left(1-\alpha_{2}\right) f\left(\bar{\rho}_{2}\right)}{f\left(\bar{\rho}_{2}\right)+\left(1-\alpha_{1}\right) f\left(\bar{\rho}_{1}\right)},
$$

(iii) $\left(S_{5}, I_{6}, S_{6}\right)$

$\alpha_{S_{5}, 6}=\frac{\alpha_{3} f\left(\bar{\rho}_{3}\right)+\left(1-\alpha_{2}\right) f\left(\bar{\rho}_{2}\right)}{f\left(\bar{\rho}_{3}\right)+\left(1-\alpha_{2}\right) f\left(\bar{\rho}_{2}\right)}, \alpha_{S_{5}, S_{6}}=\frac{\left(1-\alpha_{3}\right) f\left(\bar{\rho}_{3}\right)}{f\left(\bar{\rho}_{3}\right)+\left(1-\alpha_{2}\right) f\left(\bar{\rho}_{2}\right)}$.

A car started from $I_{1}$ reach $I_{4}$ and $I_{5}$. Then, we set $\alpha_{S 1,4}=\alpha_{1}$ and $\alpha_{S 1, S 2}=\left(1-\alpha_{1}\right)$. A car started from $I_{2}$ reach $I_{5}$ and $I_{6}$. Then, we set $\alpha_{S 3,5}=\alpha_{2}$ and $\alpha_{S 3, S 4}=\left(1-\alpha_{2}\right)$. A car started from $I_{3}$ reach $I_{6}$ and $I_{4}$. Then, we put $\alpha_{S 5,6}=\alpha_{3}$ and $\alpha_{S 5, S 6}=\left(1-\alpha_{3}\right)$. Under the given assumption, we modify in time the distribution coefficient. With such choice, one can find a time $T>0$ so that the solution is given by the flux indicated in Figure 4 hold for each $t \geq T$. This completes the proof.

4.2. Supply Limited Case. In this case, we have possible traffic jams if the constant fluxes on each road in Figure 4 do exceed the maximum flux $f\left(\rho_{c}\right)$, such that $f\left({ }^{-} \rho_{1}\right)+\left(1-\alpha_{3}\right) f\left({ }^{-} \rho_{3}\right)>f\left(\rho_{c}\right)$, $f\left({ }^{-} \rho_{2}\right)+\left(1-\alpha_{1}\right) f\left({ }^{-} \rho_{1}\right)>f\left(\rho_{c}\right)$, and $f\left({ }^{-} \rho_{3}\right)+\left(1-\alpha_{2}\right) f\left({ }^{-} \rho_{2}\right)>f\left(\rho_{c}\right)$ conditions hold. Instantaneously, shocks are produced on road networks of the roundabout at junctions $J_{1}, J_{3}$, and $J_{5}$.

Now suppose that $0<p_{i}<1, i=1,2,3$ be the priority parameter applied at merging junctions $J_{1}, J_{3}$, and $J_{5}$. Assume further that

$$
\begin{aligned}
\gamma_{1}^{\max } & =f_{1}, \quad \gamma_{2}^{\max }=f_{2}, \quad \gamma_{3}^{\max }=f_{3}, \quad \gamma_{S_{1}}^{\max }=f\left(\bar{\rho}_{1}\right)+\left(1-\alpha_{3}\right) f\left(\bar{\rho}_{3}\right) \\
& =f\left(\rho_{c}\right), \gamma_{S_{3}}^{\max }=f_{2}\left(\rho_{2}\right)+\left(1-\alpha_{1}\right) f_{1}=f\left(\rho_{c}\right) \quad \text { and } \\
\gamma_{S_{5}}^{\max } & =f_{2}\left(\rho_{2}\right)+\left(1-\alpha_{1}\right) f_{1}=f\left(\rho_{c}\right) .
\end{aligned}
$$

For simplicity, we take $a:=\left(1-\alpha_{1}\right), b:=\left(1-\alpha_{2}\right), c:=$ $\left(1-\alpha_{3}\right)$, and $f_{i}:=f\left({ }^{-} \rho_{i}\right), i=1,2,3$ to be used in the following theorem. We summarized junction wave interaction in Table 1. 
TABLE 1: Shock wave at merging junctions.

\begin{tabular}{|c|c|c|c|c|}
\hline & Derivation & Case 1 & Case 2 & Case 3 \\
\hline \multirow[t]{2}{*}{$J_{1}$} & $f_{1}+c f_{3}>f\left(\rho_{c}\right) \Rightarrow \frac{f_{1}}{f\left(\rho_{c}\right)}>1-c \frac{f_{3}}{f\left(\rho_{c}\right)}$ & $\begin{array}{c}p_{1} \leq 1-c \frac{f_{3}}{f\left(\rho_{c}\right)} \\
\widehat{\gamma}_{1}=f\left(\rho_{c}\right)-c f_{3}, \\
\widehat{\gamma}_{S_{6}}=c f_{3} .\end{array}$ & $\begin{array}{c}1-c \frac{f_{3}}{f\left(\rho_{c}\right)}<p_{1}<\frac{f_{1}}{f\left(\rho_{c}\right)} \\
\widehat{\gamma}_{1}=p_{1} f\left(\rho_{c}\right) \\
\widehat{\gamma}_{S_{6}}=\left(1-p_{1}\right) f\left(\rho_{c}\right) .\end{array}$ & $\begin{array}{c}p_{1} \geq \frac{f_{1}}{f\left(\rho_{c}\right)} \\
\widehat{\gamma}_{1}=f_{1}, \\
\widehat{\gamma}_{S_{6}}=f\left(\rho_{c}\right)-f_{1} .\end{array}$ \\
\hline & Interpretation $\rightarrow$ & Shock produced on $I_{1}$ not on $S_{6}$ & $\begin{array}{l}\text { Shock produced on } I_{1} \text { and } \\
S_{6}\end{array}$ & Shock produced on $S_{6}$ not on $I_{1}$ \\
\hline & $f_{2}+a f_{1}>f\left(\rho_{c}\right) \Rightarrow \frac{f_{2}}{f\left(\rho_{c}\right)}>1-a \frac{f_{1}}{f\left(\rho_{c}\right)}$ & $\begin{array}{c}p_{2} \leq 1-a \frac{f_{1}}{f\left(\rho_{c}\right)} \\
\widehat{\gamma}_{2}=f\left(\rho_{c}\right)-a f_{1} \\
\widehat{\gamma}_{S_{2}}=a f_{1}\end{array}$ & 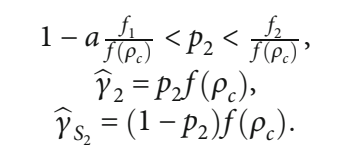 & $\begin{array}{c}p_{2} \geq \frac{f_{2}}{f\left(\rho_{c}\right)} \\
\widehat{\gamma}_{2}=f_{2}, \\
\widehat{\gamma}_{S_{2}}=f\left(\rho_{c}\right)-f_{2} .\end{array}$ \\
\hline & Interpretation $\rightarrow$ & Shock produced on $I_{2}$ not on $S_{2}$ & $\begin{array}{l}\text { Shock produced on } I_{2} \text { and } \\
\qquad S_{2}\end{array}$ & Shock produced on $S_{2}$ not on $I_{2}$ \\
\hline & $f_{3}+b f_{3}>f\left(\rho_{c}\right) \Rightarrow \frac{f_{3}}{f\left(\rho_{c}\right)}>1-b \frac{f_{2}}{f\left(\rho_{c}\right)}$ & $\begin{array}{c}p_{3} \leq 1-b \frac{f_{2}}{f\left(\rho_{c}\right)} \\
\widehat{\gamma}_{3}=f\left(\rho_{c}\right)-b f_{2} \\
\widehat{\gamma}_{S_{4}}=b f_{2} .\end{array}$ & $\begin{array}{c}1-b \frac{f_{2}}{f\left(\rho_{c}\right)}<p_{3}<\frac{f_{3}}{f\left(\rho_{c}\right)} \\
\widehat{\gamma}_{3}=p_{3} f\left(\rho_{c}\right) \\
\widehat{\gamma}_{S_{4}}=\left(1-p_{3}\right) f\left(\rho_{c}\right)\end{array}$ & $\begin{array}{c}p_{3} \geq \frac{f_{3}}{f\left(\rho_{c}\right)} \\
\widehat{\gamma}_{3}=f_{3}, \\
\widehat{\gamma}_{S_{4}}=f\left(\rho_{c}\right)-f_{3} .\end{array}$ \\
\hline & Interpretation $\rightarrow$ & Shock produced on $I_{3}$ not on $S_{4}$ & $\begin{array}{l}\text { Shock produced on } I_{3} \text { and } \\
\qquad S_{4}\end{array}$ & Shock produced on $S_{4}$ not on $I_{3}$ \\
\hline
\end{tabular}

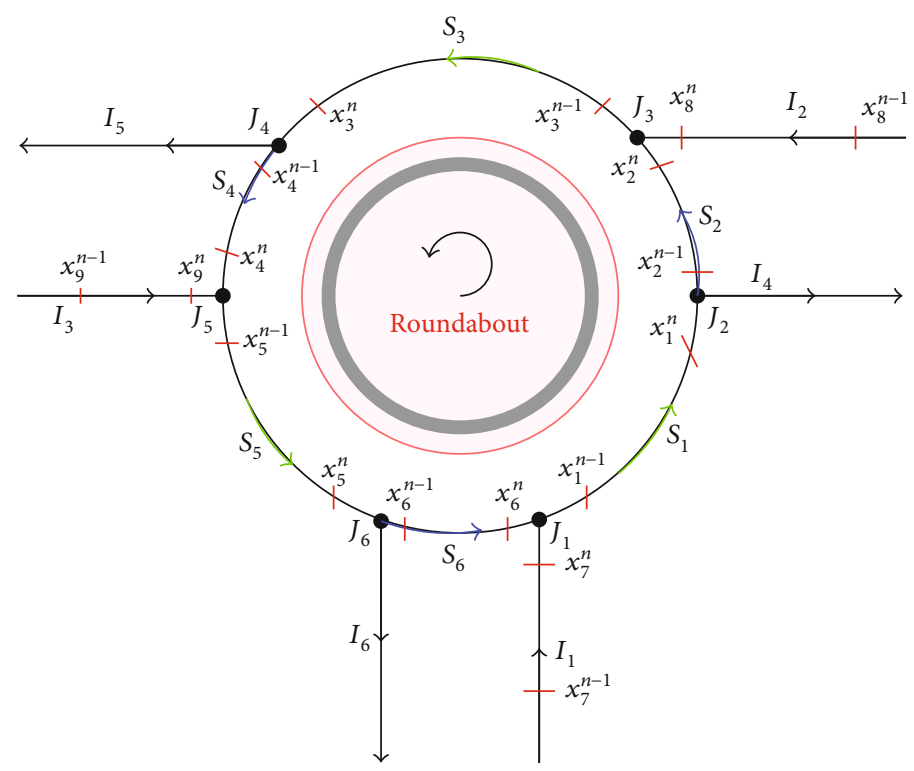

FIGURE 5: Wave junction intersections on the network of a roundabout.

Assume that we are in case 1 as given in Table 1 at junctions $J_{1}, J_{3}$, and $J_{5}$. Then, rarefaction waves are produced on roads $S_{2}, S_{4}$, and $S_{6}$. These rarefaction waves decrease the traffic density at their respective junctions, and thus, the road network of the roundabout is not congested. Thus, we have proved the follow result.

Theorem 8. Suppose that $p_{i} \in[0,1], i=1,2,3$ be priority parameters at $J_{1}, J_{3}$ and $J_{5}$ of the roundabout, respectively, such that $p_{1} \leq 1-c\left(f_{3} / f\left(\rho_{c}\right)\right), p_{2} \leq 1-a\left(f_{1} / f\left(\rho_{c}\right)\right)$ and $p_{3} \leq 1-b\left(f_{2} / f\left(\rho_{c}\right)\right)$ at these junctions, then traffic flux on the road networks of a roundabout never jammed.
Next, we give detailed analysis at each junction when the situation is fully supply limited.

This corresponds to case 2 of Table 1 at junctions $J_{1}, J_{3}$, and $J_{5}$. Since a shock is produced on all entering roads at each junctions, then rarefaction are produced on roads $S_{2}, S_{4}$, and $S_{6}$. We present this situation case by case as follows.

4.2.1. Case ( $i)$. The flux on road $S_{1}$ composed of $p_{1} f\left(\rho_{c}\right)$ from road $I_{1}$ and $\left(1-p_{1}\right) f\left(\rho_{c}\right)$ from road $S_{6}$. Since $\alpha_{1}$ of the flux from road $S_{1}$ exists through roads $I_{4}$, the part of the flux on $S_{1}$ is $\alpha_{1} p_{1} f\left(\rho_{c}\right)+\left(1-p_{1}\right) f\left(\rho_{c}\right)=\left(1-a p_{1}\right) f\left(\rho_{c}\right)$ that exits to road $I_{4}$. All flux from road $S_{6}$ leave the system through road $I_{4}$. The remaining flux $a p_{1} f\left(\rho_{c}\right)$ will proceed to road $S_{2}$. In terms of 
traffic distribution matrix, this is equivalent to saying that $\alpha_{S 21}=a p_{1}$. Since the shock wave produced on the entering road propagates back with negative speed, then the shock produced at junction $J_{3}$ reaches $J_{2}$. Thus, we have a shock on $S_{1}$. We displayed wave junction interaction in Figure 5.

The values of traffic flux on road $S_{1}$ just after the shock junction interaction are equal to

$$
\alpha_{S_{21}} x_{1}^{n}=\left(1-p_{2}\right) x_{2}^{n-1} \Rightarrow x_{1}^{n}=\frac{\left(1-p_{2}\right)}{a p_{1}} x_{2}^{n-1}
$$

where $x_{2}^{n-1}$ represents the value of the flux on road $S_{2}$ after the shock junction intersection. Also, after interaction, the traffic flux on $I_{2}$ is $x_{2}^{n}=p_{2} x_{3}^{n-1}$. All the flux from road $S_{2}$ leave the network of the roundabout through $I_{5}$.

4.2.2. Case (ii). The flux on $S_{3}$ is composed of $p_{2} f\left(\rho_{c}\right)$ from road $I_{2}$ and $\left(1-p_{2}\right)$ from road $S_{2}$. Since $\alpha_{2}$ of the flux exit the system of the roundabout through $I_{5}$, the flux that leave the network of the roundabout through $I_{5}$ equal to $\alpha_{2} p_{2} f\left(\rho_{c}\right)+\left(1-p_{2}\right) f\left(\rho_{c}\right)=\left(1-b p_{2}\right) f\left(\rho_{c}\right)$. The remaining flux $\left(1-\alpha_{2}\right) p_{2} f\left(\rho_{c}\right)$ will go to $S_{4}$. This is equivalent to saying that $\alpha_{S 43}=\left(1-\alpha_{2}\right) p_{2}$. As a consequence of backward propagation of shock on the entering road, the shock produced at junction $J_{5}$ reaches junction $J_{4}$; then, we have a shock on road $S_{4}$. On the other hand, the flux on road $S_{5}$ composed of $p_{3} f\left(\rho_{c}\right)$ from road $I_{3}$ and $\left(1-p_{3}\right) f\left(\rho_{c}\right)$ from road $S_{4}$. Therefore, the value of traffic flux on road segment $S_{3}$ just after the shock junction interaction becomes:

$$
\alpha_{S_{43}} x_{3}^{n}=\left(1-p_{3}\right) x_{4}^{n-1} \Rightarrow x_{3}^{n}=\frac{\left(1-p_{3}\right)}{b p_{2}} x_{4}^{n-1}
$$

Again, just after shock junction interaction, the traffic flux on the entering road $I_{3}$ is given by $x_{9}^{n}=p_{3} x_{5}^{n-1}$. Furthermore, the flux on road $S_{5}$ contains $p_{3} f\left(\rho_{c}\right)$ from the entering road $I_{3}$ and $\left(1-p_{3}\right) f\left(\rho_{c}\right)$ from road $S_{4}$. Thus, the flux on $S_{4}$ can be expressed as $x_{4}^{n}=\left(1-p_{3}\right) x_{5}^{n-1}$.

4.2.3. Case (iii). Similarly, the traffic flux on road $S_{5}$ composed of $p_{3} f\left(\rho_{c}\right)$ from road $I_{3}$ and $\left(1-p_{3}\right) f\left(\rho_{c}\right)$ from main road $S_{4}$. But $\alpha_{3}$ of the traffic flux from the entering road $I_{3}$ leaves the roundabout through $I_{6}$ which amounts to $\alpha_{3} p_{3} f\left(\rho_{c}\right)+\left(1-p_{3}\right) f\left(\rho_{c}\right)=\left(1-c p_{3}\right) f\left(\rho_{c}\right)$. The remaining traffic flux $c p_{3} f\left(\rho_{c}\right)$ moves to road $S_{6}$. In terms of traffic distribution matrix, this amounts to $\alpha_{S 56}=\left(1-\alpha_{3}\right) p_{3}$, with similar analysis as previous cases, the value of the traffic on road $S_{5}$ just after wave junction interactions

$$
\alpha_{S_{65}} x_{5}^{n}=\left(1-p_{1}\right) x_{6}^{n-1} \Rightarrow x_{5}^{n}=\frac{\left(1-p_{1}\right)}{c p_{3}} x_{6}^{n-1}
$$

The traffic flux on road $S_{6}$ equals to $x_{6}^{n}=\left(1-p_{1}\right) x_{1}^{n-1}$ after shock junction interaction. Similarly, on the entering road $I_{1}$, we have $x_{7}^{n}=p_{1} x_{1}^{n-1}$. Thus, the shocks are produced on the whole roads of the roundabout recursively and compactly in matrix form

$$
\bar{A}=\left(\begin{array}{ccc}
\frac{1-p_{2}}{a p_{1}} & 0 & 0 \\
0 & \frac{1-p_{3}}{b p_{2}} & 0 \\
0 & 0 & \frac{1-p_{1}}{c p_{3}}
\end{array}\right) .
$$

Since the new shock induced on the roads $S_{1}, S_{3}$, and $S_{5}$ of the roundabout, then we focus only on the matrix formed by $x_{1}^{n}, x_{3}^{n}$, and $x_{5}^{n}$ denoted by $\bar{A}$. The eigenvalue of the matrix $\bar{A}$ are

$$
\begin{aligned}
\lambda_{1}= & \frac{\left(1-p_{2}\right)}{a p_{1}}, \lambda_{2}=\frac{\left(1-p_{3}\right)}{b p_{2}}, \lambda_{3}=\frac{\left(1-p_{1}\right)}{c p_{3}} \text { and } \lambda_{1} \lambda_{2} \lambda_{3} \\
= & \frac{\left(1-p_{1}\right)\left(1-p_{2}\right)\left(1-p_{3}\right)}{a b c p_{1} p_{2} p_{3}} .
\end{aligned}
$$

Moreover, at junctions $J_{2}, J_{4}$, and $J_{6}$, respectively, we can observe that

$\left(1-p_{2}\right) f\left(\rho_{c}\right)<a f_{1},\left(1-p_{3}\right) f\left(\rho_{c}\right)<b f_{2}$ and $\left(1-p_{1}\right) f\left(\rho_{c}\right)<c f_{3}$.

Furthermore, from

$$
\left\{\begin{array}{l}
1-c \frac{f_{3}}{f\left(\rho_{c}\right)}<p_{1}<\frac{f_{1}}{f\left(\rho_{c}\right)} \Rightarrow p_{1} f\left(\rho_{c}\right)<f_{1}, \\
1-a \frac{f_{1}}{f\left(\rho_{c}\right)}<p_{2}<\frac{f_{2}}{f\left(\rho_{c}\right)} \Rightarrow p_{2} f\left(\rho_{c}\right)<f_{2}, \\
1-b \frac{f_{2}}{f\left(\rho_{c}\right)}<p_{3}<\frac{f_{3}}{f\left(\rho_{c}\right)} \Rightarrow p_{3} f\left(\rho_{c}\right)<f_{3} .
\end{array}\right.
$$

From equations (30) and (31), we obtain

$$
\begin{cases}\left(1-p_{2}\right) f\left(\rho_{c}\right)<a f_{1}, & p_{1} f\left(\rho_{c}\right)<f_{1}, \\ \left(1-p_{3}\right) f\left(\rho_{c}\right)<b f_{2}, & p_{2} f\left(\rho_{c}\right)<f_{2}, \\ \left(1-p_{1}\right) f\left(\rho_{c}\right)<c f_{3}, & p_{3} f\left(\rho_{c}\right)<f_{3} .\end{cases}
$$

Simplifying, we get

$$
\frac{p_{1}}{\left(1-p_{2}\right)}<\frac{1}{a}, \frac{p_{2}}{\left(1-p_{3}\right)}<\frac{1}{b}, \text { and } \frac{p_{3}}{\left(1-p_{1}\right)}<\frac{1}{c}
$$

Since all are positive quantities, multiplication does not influence the inequalities. That is,

$$
\frac{p_{1} p_{2} p_{3}}{\left(1-p_{1}\right)\left(1-p_{2}\right)\left(1-p_{3}\right)}<\frac{1}{a b c} \Rightarrow \frac{a b c p_{1} p_{2} p_{3}}{\left(1-p_{1}\right)\left(1-p_{2}\right)\left(1-p_{3}\right)}<1
$$




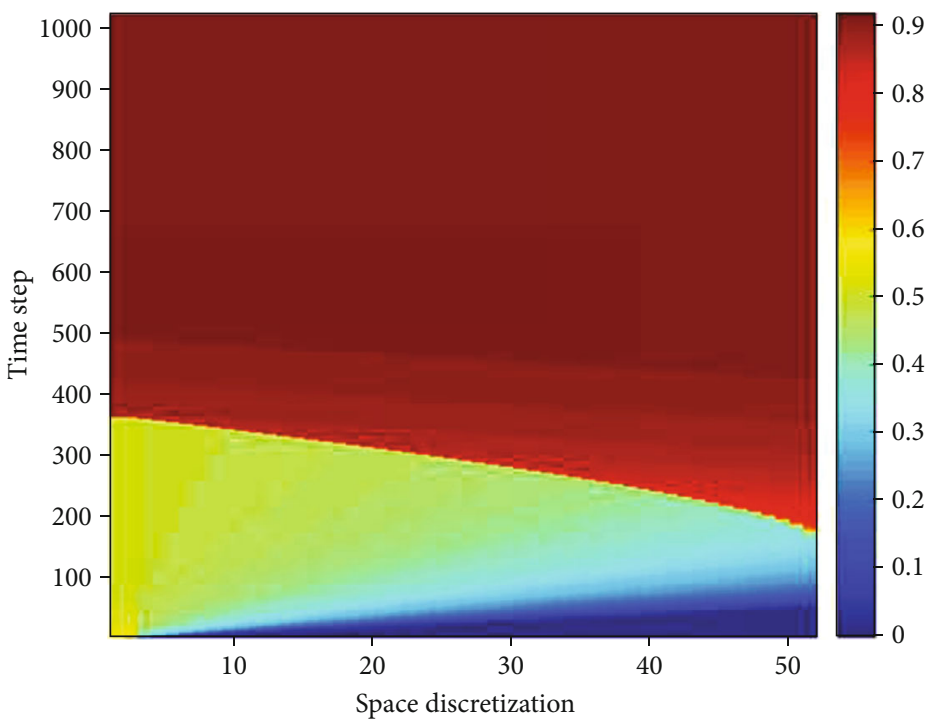

(a) Density on the entrances road

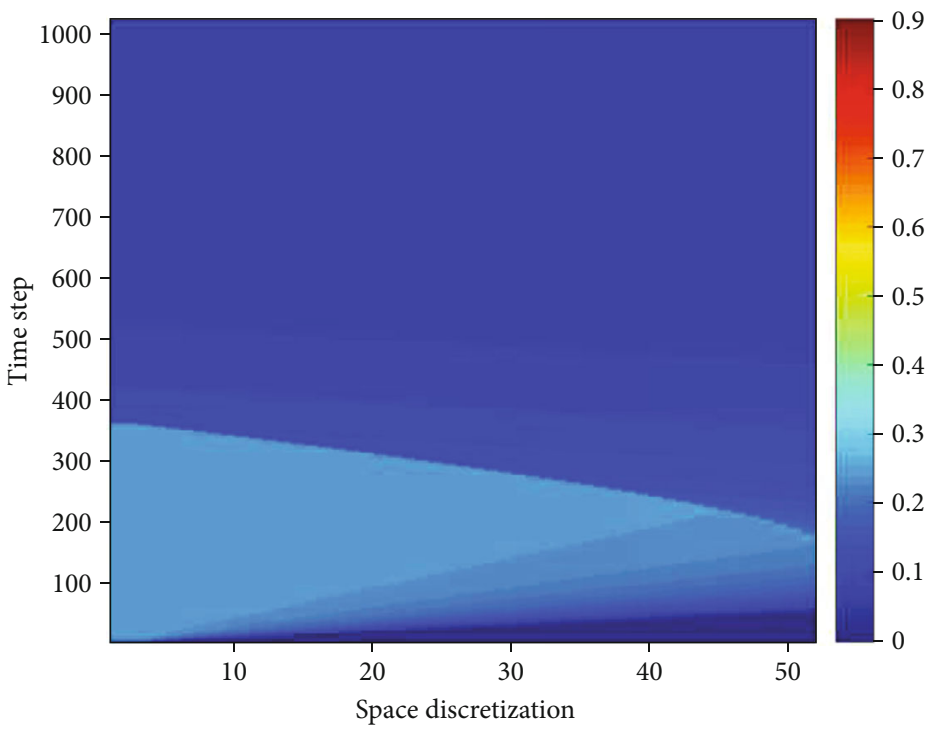

(b) Flux on the entrances road

Figure 6: Continued. 


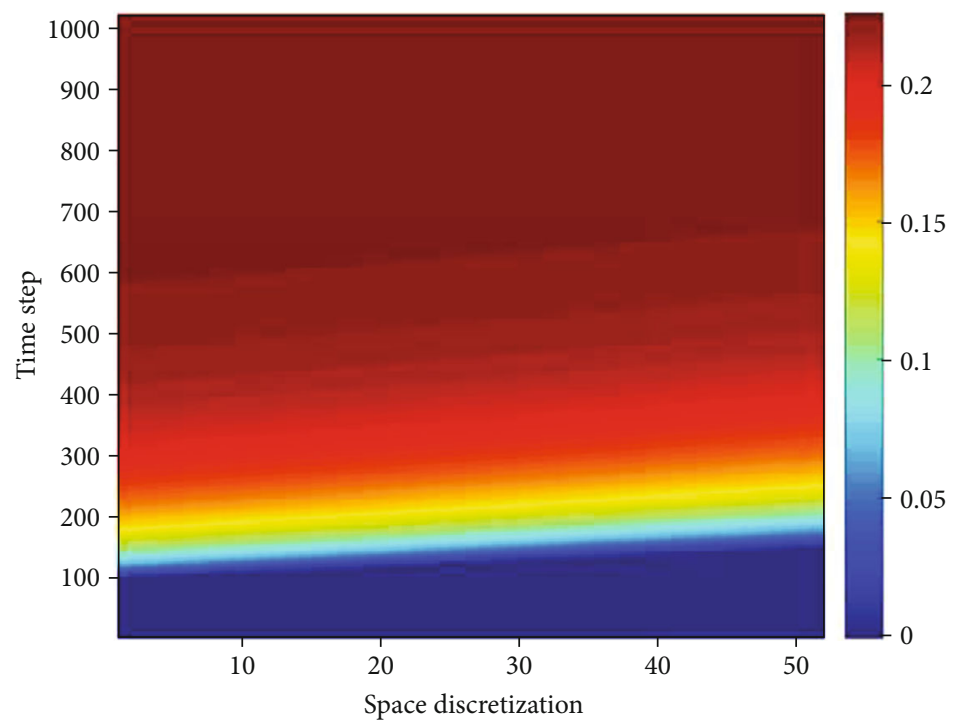

(c) Density between the diverging and merging main lane of the roundabout

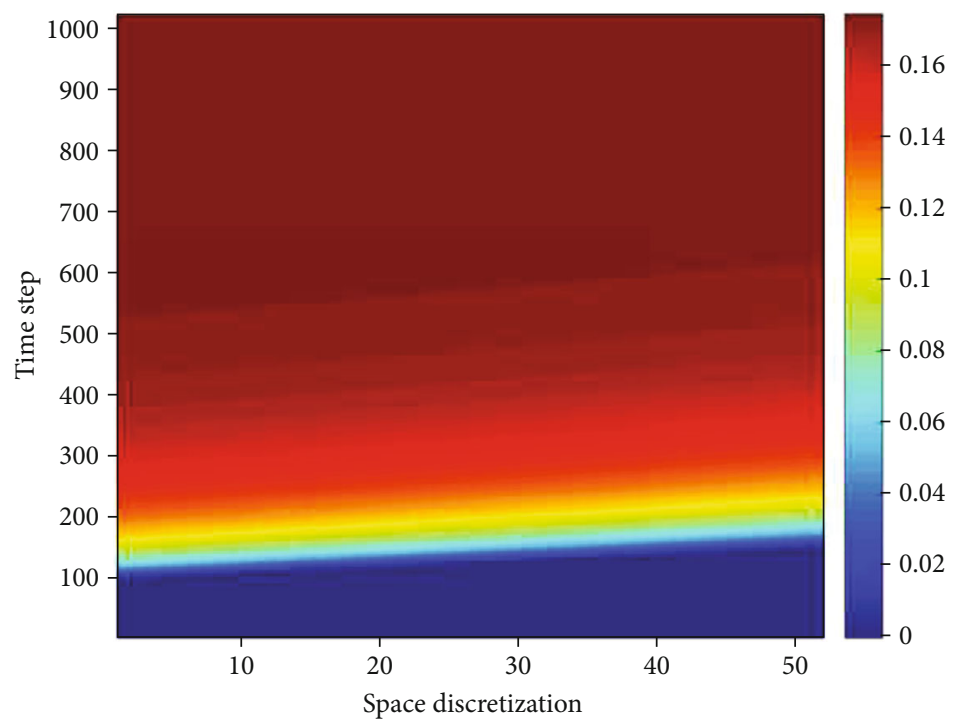

(d) Flux between the diverging and merging main lane of the roundabout

FIGURE 6: Density and flux profiles on the entrance and main lane of a roundabout in case 1. In case (a), shock waves are produced on roads $I_{1}$ , $I_{2}$, and $I_{3}$, and rarefaction waves are created on roads $S_{2}, S_{4}$, and $S_{6}$ as shown in (c) with corresponding fluxes displayed in (b) and (d), respectively.

Equivalently,

$$
\frac{\left(1-p_{1}\right)\left(1-p_{2}\right)\left(1-p_{3}\right)}{a b c p_{1} p_{2} p_{3}}>1
$$

From traffic point of view, $p_{1} f\left(\rho_{c}\right)<f_{1}, p_{2} f\left(\rho_{c}\right)<f_{2}$, and $p_{3} f\left(\rho_{c}\right)<f_{3}$ suggest that flow is congested but not jammed. That means, $\gamma_{1}^{\max }=f_{1}, \gamma_{2}^{\max }=f_{2}$, and $\gamma_{3}^{\max }=f_{3}$, as illustrated in case 2 of $J_{1}, J_{3}$, and $J_{5}$. Even though excess traffic flux on the entering roads, the roundabout is not stuck. Thus, we proved the following result.

\section{Proposition 9. Assume that}

$c\left(f_{3} / f\left(\rho_{c}\right)\right)<p_{1}<f_{1} / f\left(\rho_{c}\right), 1-a\left(f_{1} / f\left(\rho_{c}\right)\right)<p_{2}<f_{2} / f($ $\left.\rho_{c}\right)$ and $1-b\left(f_{2} / f\left(\rho_{c}\right)\right)<p_{3}<f_{3} / f\left(\rho_{c}\right)$ are satisfied for $\alpha_{1}, \alpha_{2}$ ,$\alpha_{3}$ and for all the right of way parameters $p_{1}, p_{2}, p_{3}$. If

$$
\frac{\left(1-p_{1}\right)\left(1-p_{2}\right)\left(1-p_{3}\right)}{a b c p_{1} p_{2} p_{3}}>1
$$

then the traffic flow on the roundabout does not stuck.

Proposition 10. If

$f_{1}=p_{1} f\left(\rho_{c}\right), f_{2}=p_{2} f\left(\rho_{c}\right), f_{3}=p_{3} f\left(\rho_{c}\right)$, and $\frac{\left(1-p_{1}\right)\left(1-p_{2}\right)\left(1-p_{3}\right)}{a b c p_{1} p_{2} p_{3}}=1$,

then the traffic flow on the roundabout is not jammed. 


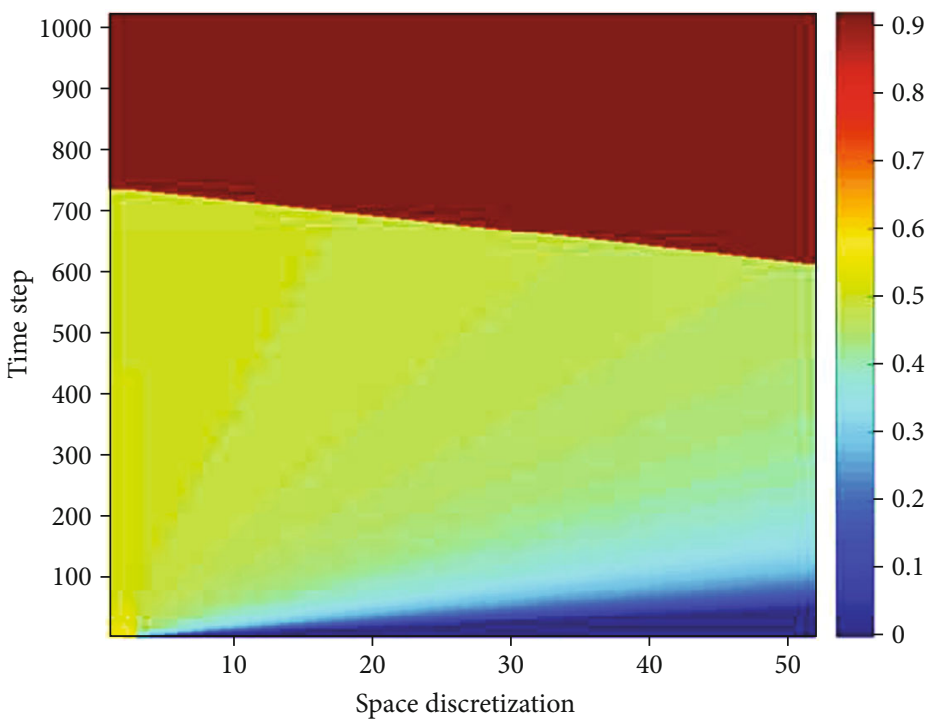

(a) Density on the entrances road in case 2

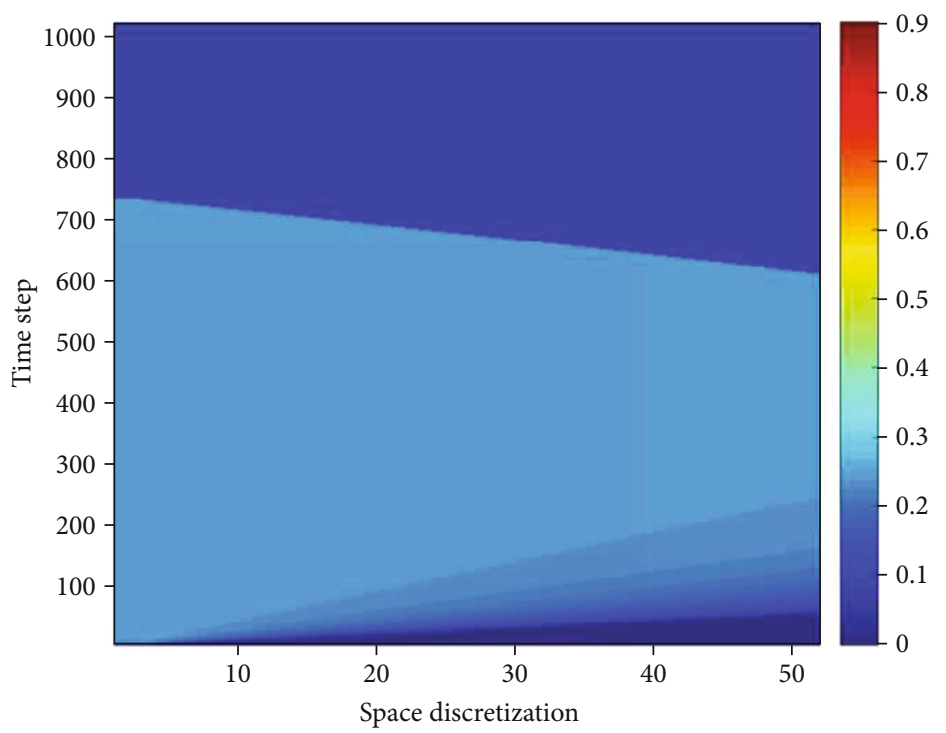

(b) Flux on the entrances road in case 2

Figure 7: Continued. 


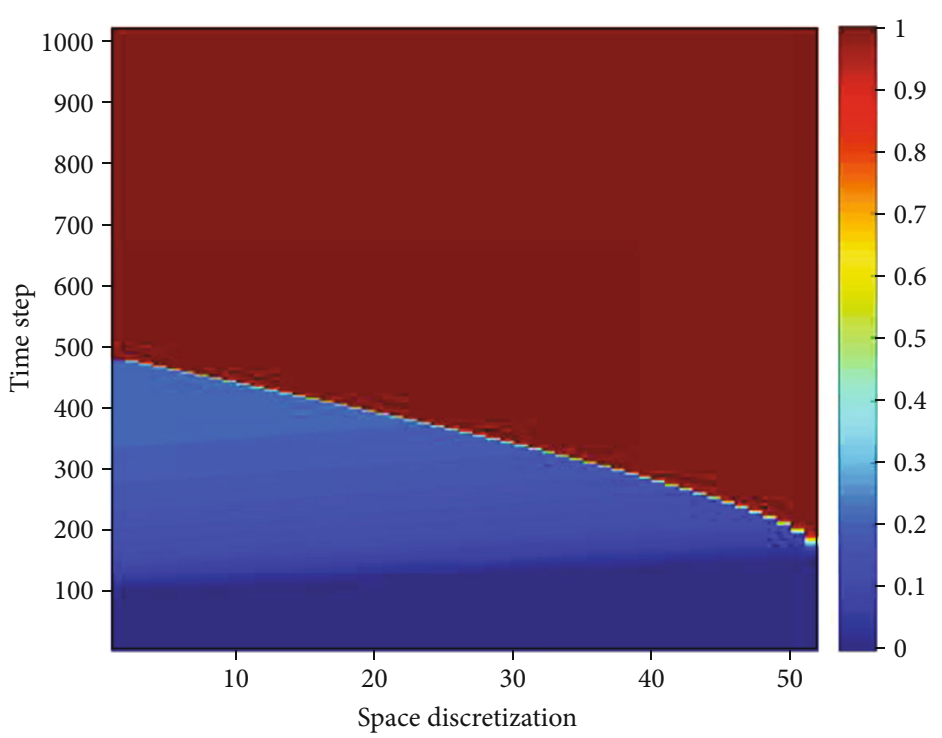

(c) Density between the diverging and merging main lane of the roundabout in case 2

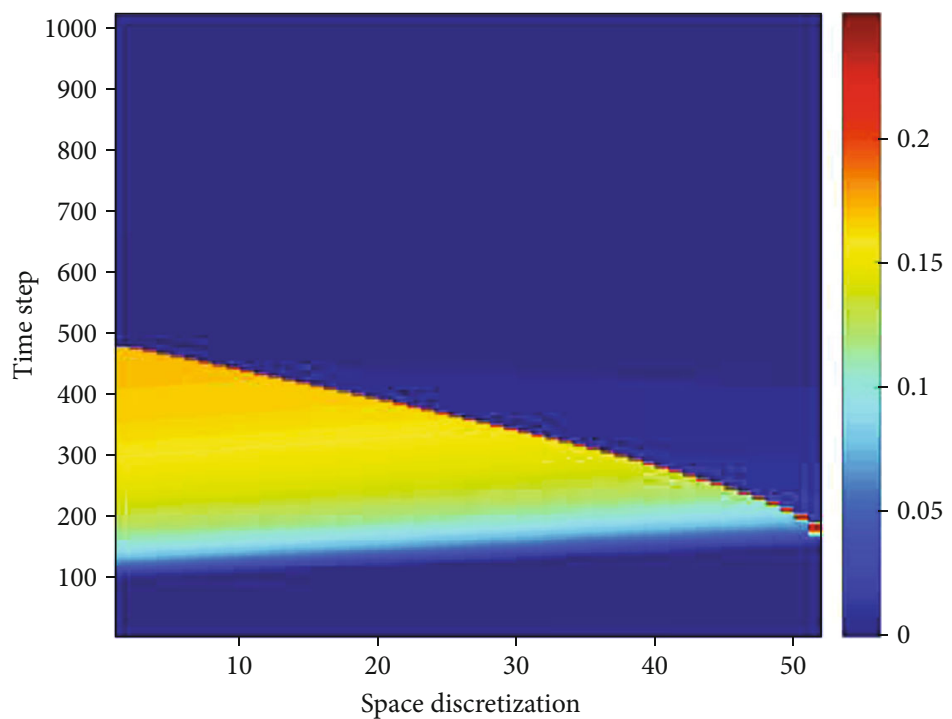

(d) Flux between the diverging and merging main lane of the roundabout in case 2

FIGURE 7: Density and flux profiles on the entrance and main lane of a roundabout in case 2. Due to traffic congestion, shock waves are produced on roads $\left(I_{1}, I_{2}, I_{3}\right)$ in the case of (a) and on $\left(S_{2}, S_{4}, S_{6}\right)$ in the case of (c) with corresponding fluxes as shown in (b) and (d), respectively.

Proof. From case (iii) we know that $c f_{3}=f\left(\rho_{c}\right)-f_{1}$, since $f_{1}$ $=p_{1} f\left(\rho_{c}\right)$. Then, $c f_{3}=f\left(\rho_{c}\right)-p_{1} f\left(\rho_{c}\right)=\left(1-p_{1}\right) f\left(\rho_{c}\right)$. On the other hand, traffic flux on $S_{1}$ equals $f_{1}+c f_{3}=f\left(\rho_{c}\right) \Leftrightarrow$ $p_{1} f\left(\rho_{c}\right)+\left(1-p_{1}\right) f\left(\rho_{c}\right)=f\left(\rho_{c}\right)$, similar for other cases. This implies that all the traffic flux are entered the junction smoothly. Thus, it attains maximum flow.

Finally, we consider case 3 given Table 1 . If the strict inequality hold at all junctions $J_{1}\left(I_{1}, S_{6}, S_{1}\right), J_{3}\left(I_{2}, S_{2}, S_{3}\right)$, and $J_{5}\left(I_{3}, S_{4}, S_{5}\right)$, then rarefactions are produced on the roads $S_{1}, S_{3}$, and $S_{5}$ while shocks are produced on roads $S_{2}$, $S_{4}$, and $S_{6}$.

Proposition 11. If $p_{i}>f_{i} / f\left(\rho_{c}\right), i=1,2,3$, then the traffic flux on the roundabout is completely jammed.
Proof. Following the arrangement given under case 2, we get the following inequalities:

$$
\begin{array}{ll}
\left(1-p_{2}\right) f\left(\rho_{c}\right)<a f_{1}, & p_{1} f\left(\rho_{c}\right)>f_{1}, \\
\left(1-p_{3}\right) f\left(\rho_{c}\right)<b f_{2}, & p_{2} f\left(\rho_{c}\right)>f_{2}, \\
\left(1-p_{1}\right) f\left(\rho_{c}\right)<c f_{3}, & p_{3} f\left(\rho_{c}\right)>f_{3} .
\end{array}
$$

This is the same as saying

$$
\left(1-p_{2}\right) f\left(\rho_{c}\right)<a p_{1} f\left(\rho_{c}\right), \quad\left(1-p_{3}\right) f\left(\rho_{c}\right)<b p_{2} f\left(\rho_{c}\right),
$$$$
\text { and }\left(1-p_{1}\right) f\left(\rho_{c}\right)<c p_{3} f\left(\rho_{c}\right) \text {. }
$$ 


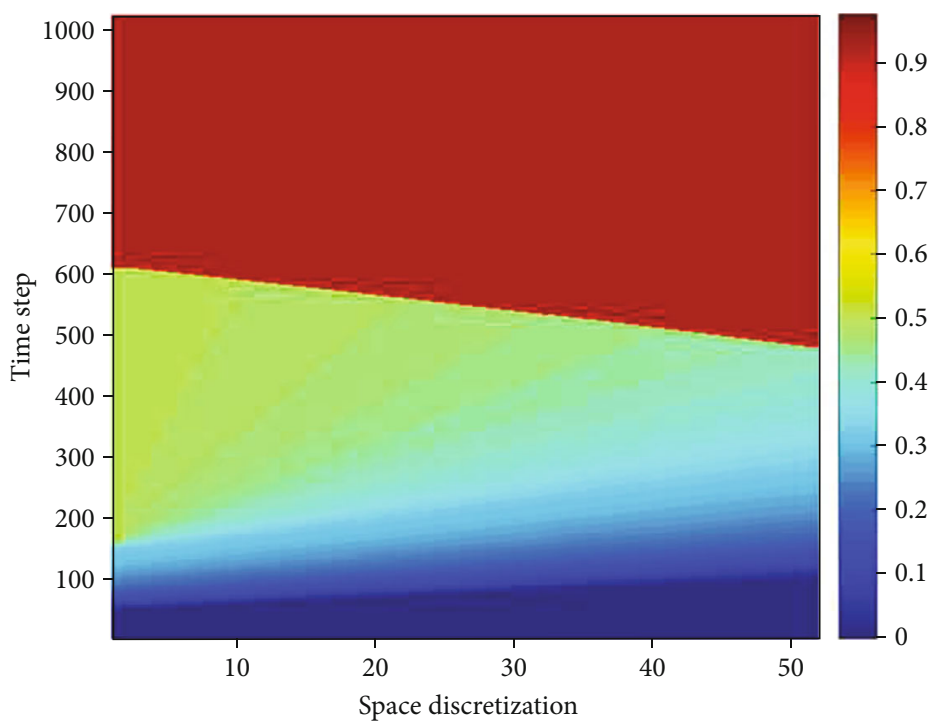

(a) Density between the merging and diverging main lane of the roundabout

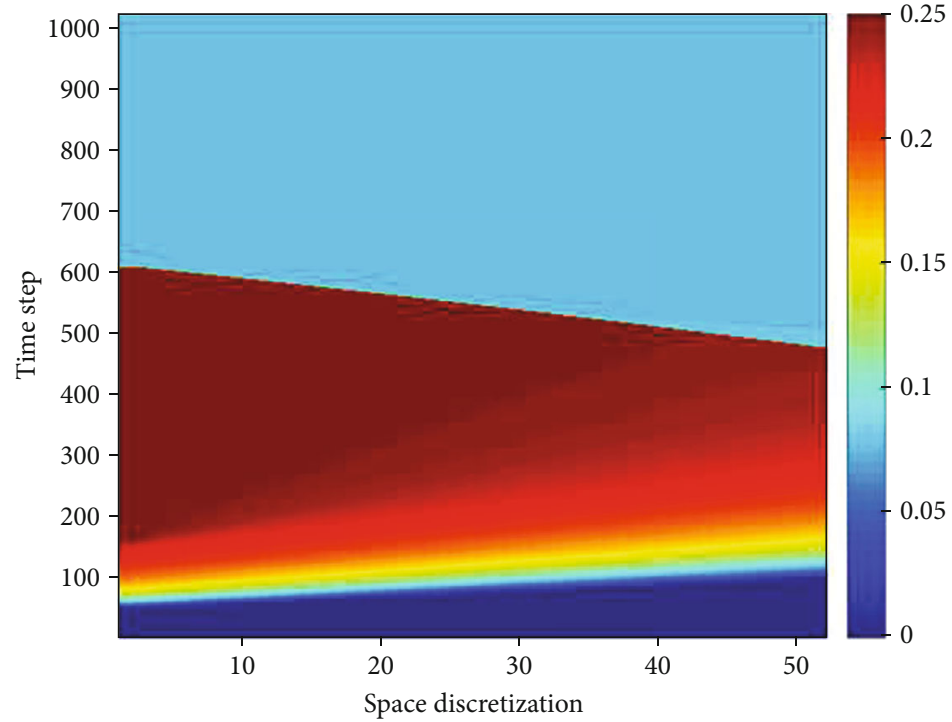

(b) Flux between the merging and diverging main lane of the roundabout

Figure 8: Continued. 


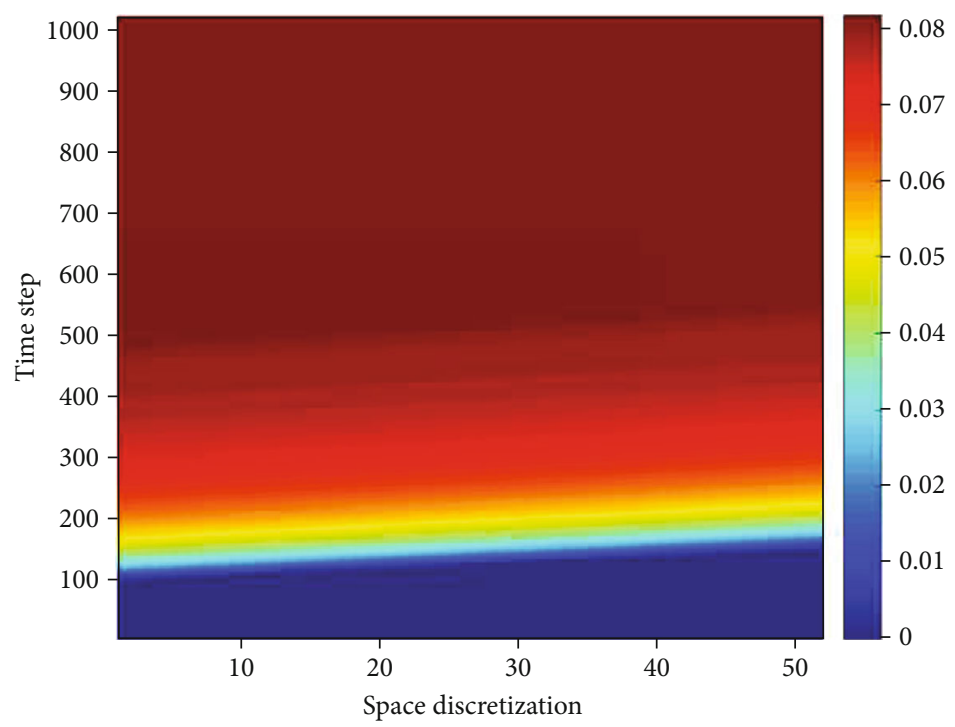

(c) Density on the exits road

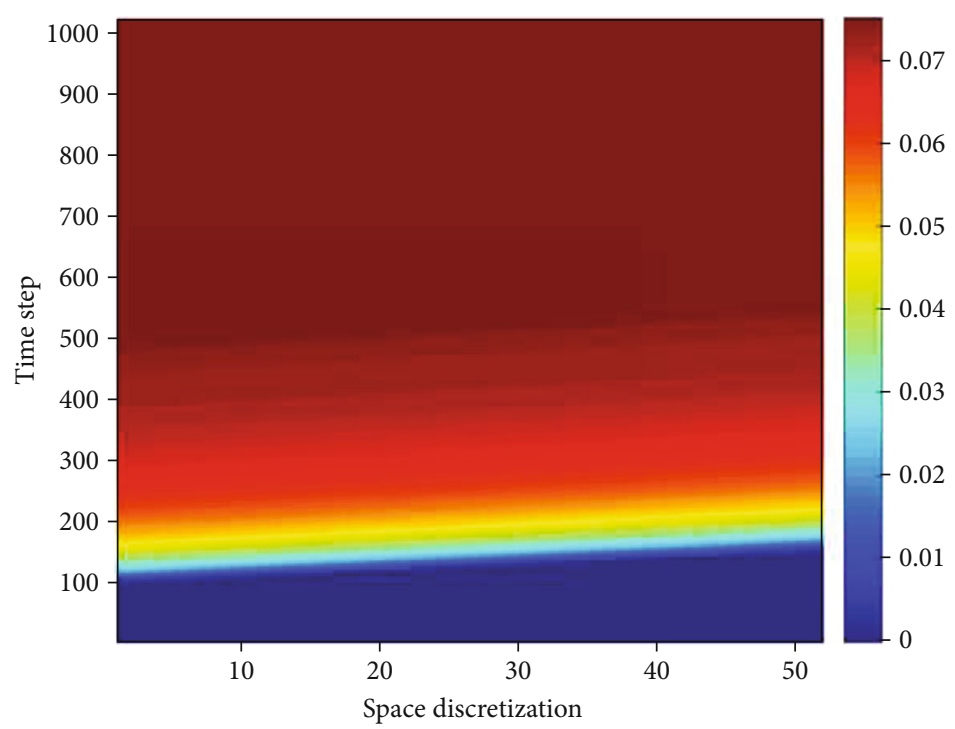

(d) Flux on the exits road

FIgURE 8: Density and flux profiles between the merging and diverging main lane of the roundabout under consideration and on the existing roads. In the case (a), shocks are produced on roads $S_{1}, S_{3}$, and $S_{5}$. Further, rarefaction waves are produced on roads $I_{4}, I_{5}$, and $I_{6}$ as shown in (c). Corresponding traffic fluxes are shown in (b) and (d), respectively.

From this, we conclude that

$\left(1-p_{1}\right)\left(1-p_{2}\right)\left(1-p_{3}\right)<a b c p_{1} p_{2} p_{3} \Rightarrow \frac{\left(1-p_{1}\right)\left(1-p_{2}\right)\left(1-p_{3}\right)}{a b c p_{1} p_{2} p_{3}}<1$.

\section{Numerical Simulations}

In this section, we present numerical scheme used to solve problem given in (1). We define a numerical grid in $(0, T)$ $\times \mathbb{R}$ using the following notation: $\Delta x$ is the fixed grid space, $\Delta t$ is the time step given by the CFL condition and $\left(t^{n}, x_{j}\right)$ $=(n \Delta t, j \Delta x)$ for $n \in \mathbb{N}$, and $j \in \mathbb{Z}$ are the grid points. Each road is divided into $N+1$ cells numbered from 0 to $N$. The first and last cells of an edge are always a junction, and we assume that these cells are ghost cells. The scheme used for solving equation (1) is the Godunov scheme as introduced in $[20,23]$ and it is based on exact solutions to the Riemann problem. The main idea of this method is to approximate the initial datum by a piecewise constant function; then, the corresponding Riemann problems are solved exactly, and a global solution is obtained by piecing them together. Under the CFL (Courant-Friedrichs-Lewy) condition, it holds:

$$
\Delta t \max _{j \in \mathbb{Z}}\left|\lambda_{j+\frac{1}{2}}^{n}\right| \leq \Delta x
$$




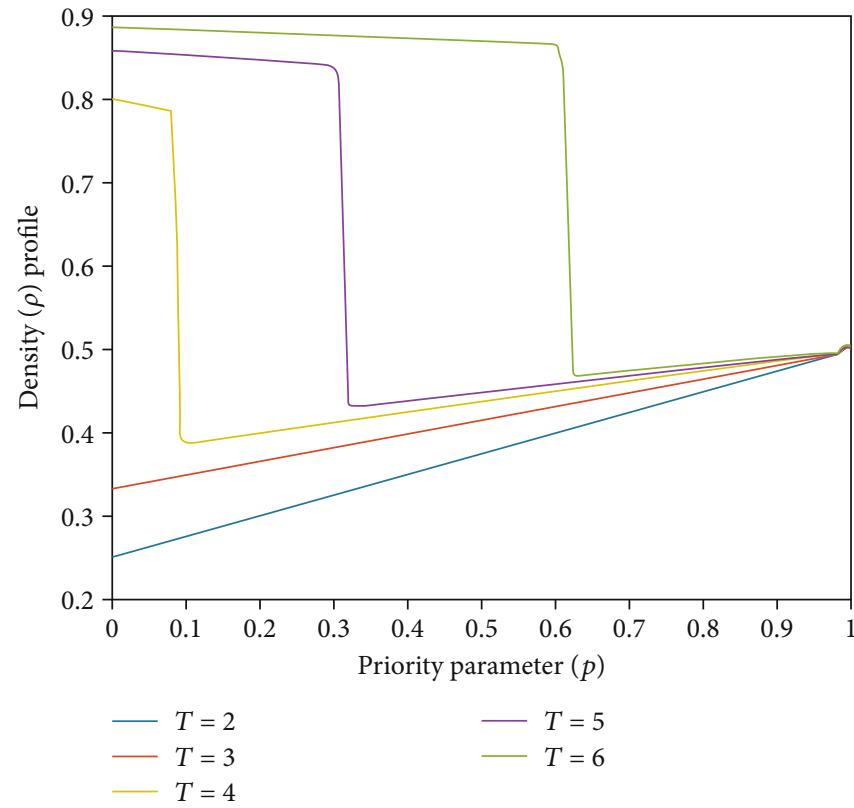

(a) Density profile on the entrance road of a roundabout when priority is given to entering traffic

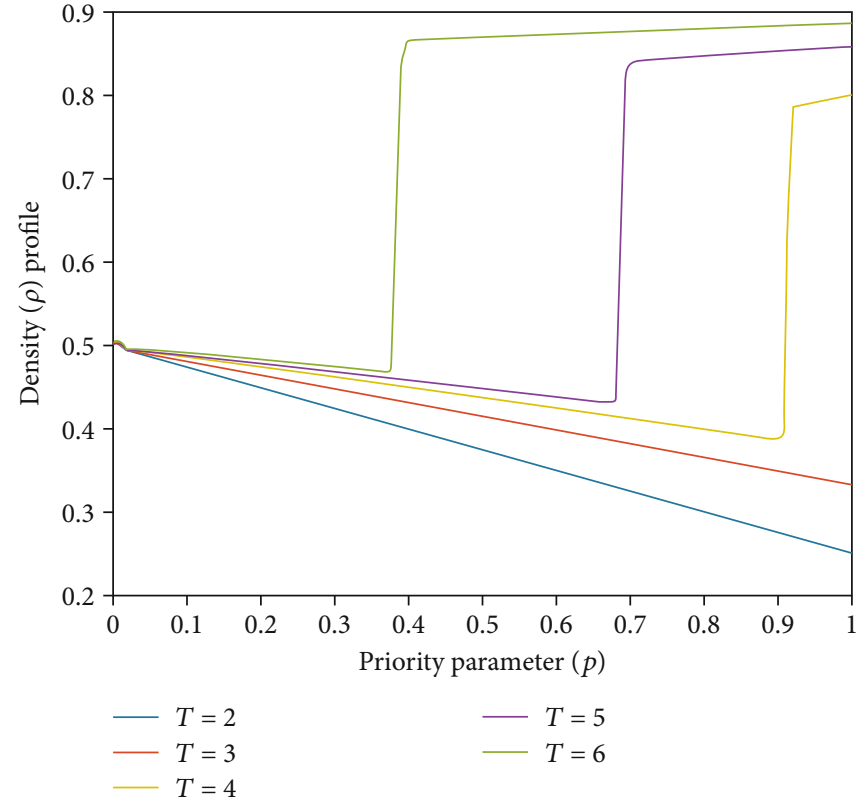

(b) Density profile on the entrance road of a roundabout when priority is given to traffic on the main lane of a roundabout

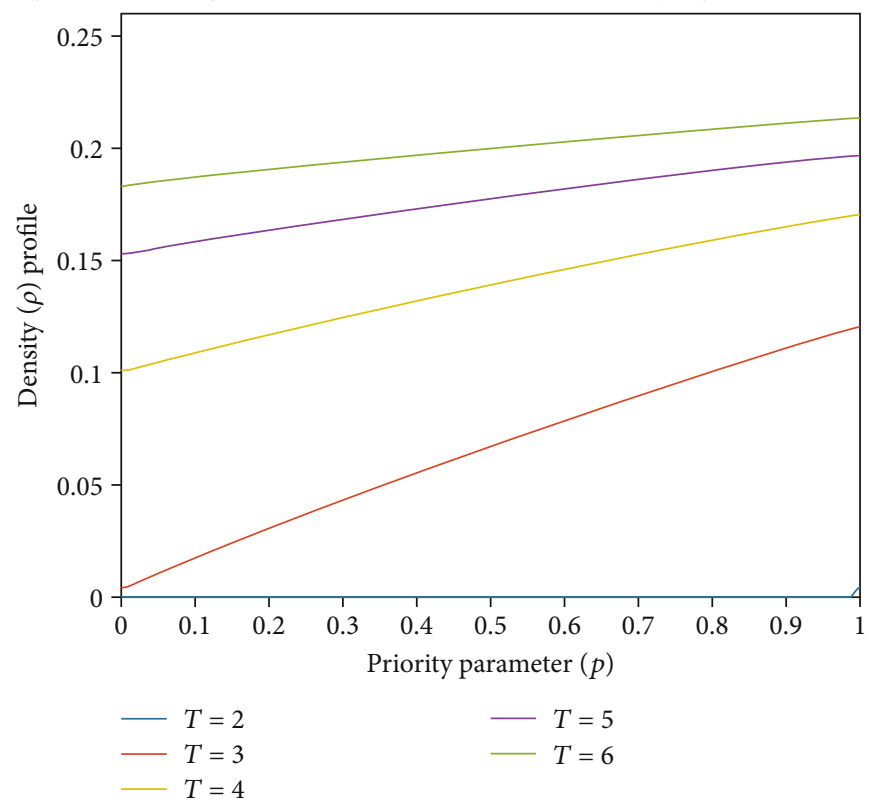

(c) Density profile on the main lane of a roundabout when priority is given to entering traffic

Figure 9: Continued. 


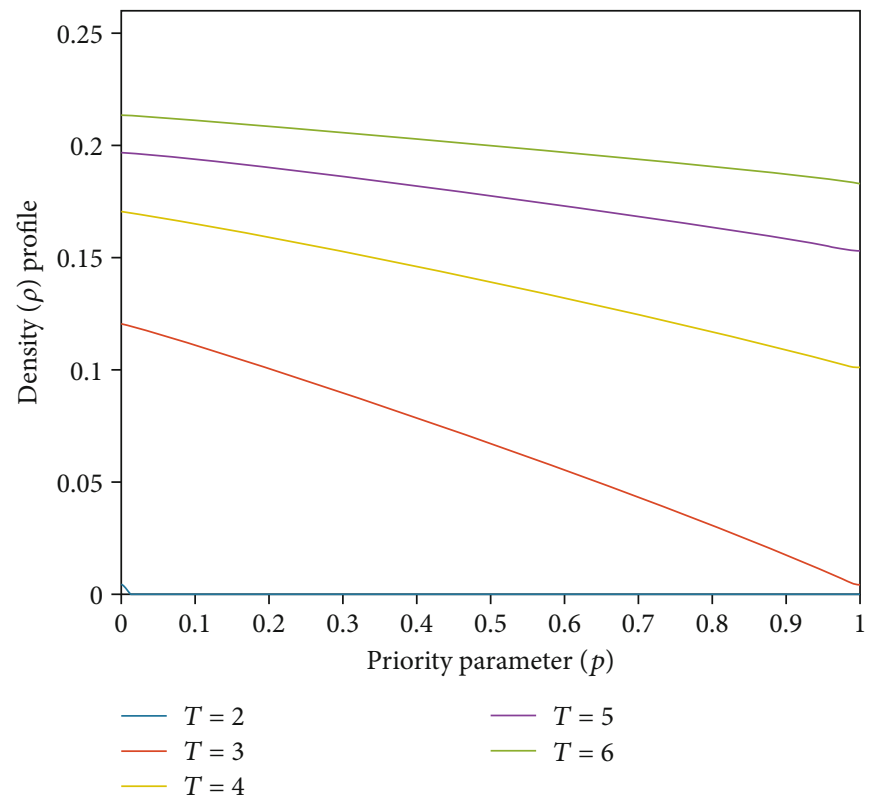

(d) Density profile on the main lane of a roundabout when priority is given to traffic on the main lane itself

FIGURE 9: Evolution of density profiles on the entrance road and main lane of a roundabout versus priority parameters for time simulation $T$ between $2 \leq T \leq 6$.

where $\lambda_{j+1 / 2}^{n}$ is the speed of the wave of the Riemann problem solution at the interface $x_{j+1 / 2}$ at the time $t^{n}$, the numerical scheme can be written as

$$
\rho_{j}^{n+1}=\rho_{j}^{n}-\frac{\Delta t}{\Delta x}\left(F\left(\rho_{j}^{n}, \rho_{j+1}^{n}\right)-F\left(\rho_{j-1}^{n}, \rho_{j}^{n}\right)\right)
$$

where the numerical flux $F$ for a concave flux function is given by

$$
F(u, v)=\left\{\begin{array}{l}
\min f(u, v) \text { if } u \leq v, \\
f(u) \text { if } v<u<\rho_{c} \\
f^{\max } \text { if } v<\rho_{c}<u, \\
f(v) \text { if } \rho_{c}<v<u .
\end{array}\right.
$$

5.1. Boundary Conditions. Each road is divided in $N+1$ cells numbered from 0 to $N$. For the entering roads, we proceed by setting:

$$
\rho_{0}^{n+1}=\rho_{0}^{n}-\frac{\Delta t}{\Delta x}\left[F\left(\rho_{0}^{n}, \rho_{0}^{n}\right)-F\left(\rho_{0}^{n}, \rho_{1}^{n}\right)\right]
$$

while for the exiting ones, we set

$$
\rho_{N}^{n+1}=\rho_{N}^{n}-\frac{\Delta t}{\Delta x}\left[F\left(\rho_{N-1}^{n}, \rho_{N}^{n}\right)-F\left(\rho_{N}^{n}, \rho_{N}^{n}\right)\right] .
$$

5.2. Conditions at the Junctions. From the entering roads which are connected at the junction at the right endpoint, we set

$$
\rho_{N}^{n+1}=\rho_{N}^{n}-\frac{\Delta t}{\Delta x}\left[\widehat{\gamma}_{\text {in }}-F\left(\rho_{N-1}^{n}, \rho_{N}^{n}\right)\right],
$$

while for the exiting ones, connected at the junctions at the left point, we set

$$
\rho_{0}^{n+1}=\rho_{0}^{n}-\frac{\Delta t}{\Delta x}\left[F\left(\rho_{0}^{n}, \rho_{1}^{n}\right)-\widehat{\gamma}_{\text {out }}\right] .
$$

5.3. Simulation Results. For simulation purpose, we chose a concave fundamental diagram as introduced in equation (3) with the following values for parameters: $v_{\max }=1 ; \rho_{\max }=1$ $; L=50 ; \rho_{c}=0.5 ; T=20, \Delta x=0.0196, \Delta t=0.0196$. We assume that at initial time $t=0$ all the roads are empty and influx at boundary of entering roads are equal to 0.9 . In the case of demand limited, the traffic evolution is governed by conservation law and the splitting rate to describe how traffic coming from the entering roads choose to distributed to their corresponding intermediate roads and the external exiting roads. Thus, in this case, rarefaction wave fill the portion of the roads of the roundabout. But, in the supply limited case, the traffic congestion can occur at merging junctions and shock wave propagating back, which is the one we simulated in the results.

The results obtained are the evolution of density and flux on entrance roads, on exit roads, roads between merging and diverging junctions, and roads between diverging and merging junctions versus space discretization at different time steps as shown in Figures 6-8. Figure 9 displays the evolution of traffic density profiles versus priority parameters. In these figures, different colors show different traffic scenarios over simulation time. The blue color corresponds to demand limited case while the red color denotes congested state with 
value indicated on the color bars. In Figure 6, we illustrate the simulation result corresponds to case 1 at merging junctions $J_{1}, J_{3}$, and $J_{5}$.

Similar to case 1, we performed numerical simulation for case 3. In this case, shock waves are produced on the roads $S_{2}, S_{4}$, and $S_{6}$ propagating back while rarefaction waves on $I_{1}, I_{2}$, and $I_{3}$ propagating forward in time. In the next, we describe the simulation results for case 2. In this case, the roundabout under consideration is fully supply limited case as shown in Figures 7 and 8. The shock wave occurred on the entrance roads $\left(I_{1}, I_{2}, I_{3}\right)$, and between diverging and merging junctions of roads $\left(S_{2}, S_{4}, S_{6}\right)$ is depicted in Figure 7. As a result, backward propagating shock wave produced between merging and diverging junctions, that is, on the roads $\left(S_{1}, S_{3}, S_{5}\right)$ as displayed in Figure 8(a).

Figure 9 shows the evolution of traffic profiles on the entrance road and main roads of a roundabout versus priority parameters for simulation time $T$ between $2 \leq T \leq 6$. The impact of priority parameters on the external entrance roads is shown in Figure 9(a), and on the main lanes of the roundabout are also displayed in Figure 9(b). Figures 9(c) and 9(d) present density profile on the main lane of a roundabout when priority is given to entering traffic and when priority is also given to traffic on the main lane itself, respectively.

\section{Conclusion}

In this paper, we presented the analysis of traffic evolution on road network of roundabout with three entering and three exiting roads. The flow of traffic on the whole road network of the roundabout is governed by nonlinear scalar hyperbolic conservation laws or the LWR model. We used traffic distribution matrix at diverging and priority parameters at merging junctions. Also, we investigated the performance of a roundabout in regulating traffic evolution both in the case of demand and supply limited cases with details mathematical analysis. In demand limited case, the number of cars reaching the roundabout is less than the optimal capacity of the roundabout. Thus, the junction stays demand limited, and we did not need priority rule in that situation. The traffic evolution is only governed by hyperbolic conservation laws. However, we assigned traffic distribution ratio to express how traffic coming from the entering roads choose to distributed to their corresponding intermediate roads and the external exiting roads. Since the number of cars reaching the roundabout was greater than the optimal capacity of the roundabout in supply limited case, we have been used priority rule at merging junctions. We solved the problem using the Godunov scheme. Finally, we presented results of simulations of traffic evolution on road network in terms of density and flux versus space discretization at different time steps. The simulation result indicated that rarefaction waves fill the portion of the roads of the roundabout in demand limited case, and the traffic congestion occurs at merging junctions and shock wave propagating back in supply limited case (congested state) which reduced the efficiency of a roundabout in controlling traffic flow problem. The experiment with field study and validation of the model with real data will be considered in the future work.

\section{Data Availability}

The current study does not involve any data.

\section{Disclosure}

The contents of this paper reflect the views of the authors' contribution to prepare, develop, and carry out this manuscript.

\section{Conflicts of Interest}

We, the authors of the research article entitled "Analysis of Traffic Evolution on Road Networks of a Roundabout," declare that there is no conflicts of interest in this article.

\section{Acknowledgments}

This research work was supported by the Hawassa University and Wolaita Sodo University under the Ministry of Education.

\section{References}

[1] M. J. Lighthill and G. B. Whitham, "On kinematic waves II. A theory of traffic flow on long crowded roads," Proceedings of the Royal Society of London. Series A. Mathematical and Physical Sciences, vol. 229, no. 1178, pp. 317-345, 1955.

[2] P. I. Richards, "Shock waves on the highway," Operations Research, vol. 4, no. 1, pp. 42-51, 1956.

[3] M. Burger, S. G. Ttlich, and T. Jung, "Derivation of a first order traffic flow model of Lighthill-Whitham-Richards type," IFAC-PapersOnLine, vol. 51, no. 9, pp. 49-54, 2018.

[4] J. Fang, H. Ye, and S. M. Easa, "Modified traffic flow model with connected vehicle microscopic data for proactive variable speed limit control," Journal of Advanced Transportation, vol. 2019, Article ID 8151582, 11 pages, 2019.

[5] M. Herty, S. Moutari, and G. Visconti, "Macroscopic modeling of multilane motorways using a two-dimensional secondorder model of traffic flow," SIAM Journal on Applied Mathematics, vol. 78, no. 4, pp. 2252-2278, 2018.

[6] T. Hundesa, L. Lemecha, and P. R. Koya, "One-dimensional explicit tolesa numerical scheme for solving first order hyperbolic equations and its application to macroscopic traffic flow model," Applied Mathematics, vol. 10, no. 3, pp. 119-137, 2019.

[7] N. Laurent-Brouty, G. Costeseque, and P. Goatin, "A coupled PDE-ODE model for bounded acceleration in macroscopic traffic flow models. ifac-papersonline," IFAC-PapersOnLine, vol. 51, no. 9, pp. 37-42, 2018.

[8] A. Rey, W.-L. Jin, and S. G. Ritchie, “An extension of Newell's simplified kinematic wave model to account for first-in-firstout violation: with an application to vehicle trajectory estimation," Transportation Research Part C: Emerging Technologies, vol. 109, pp. 79-94, 2019.

[9] X. Xiao and H. Duan, "A new grey model for traffic flow mechanics," Engineering Applications of Artificial Intelligence, vol. 88, article 103350, 2020.

[10] G. M. Coclite, M. Garavello, and B. Piccoli, "Traffic flow on a road network," SIAM Journal on Mathematical Analysis, vol. 36, no. 6, pp. 1862-1886, 2005. 
[11] M. Garavello and B. Piccoli, Traffic flow on Networks, Volume 1, Springfield: American institute of mathematical sciences, 2006.

[12] Z. Dong, C. Yu, D. Jitamitra, H. Y. K. Lau, and S. Srivathsan, "A time-space network flow approach to dynamic repositioning in bicycle sharing systems," Transportation Research Part B: Methodological, vol. 103, pp. 188-207, 2017.

[13] R. X. Zhong, Y. P. Huang, C. Chen, W. H. K. Lam, D. B. Xu, and A. Sumalee, "Boundary conditions and behavior of the macroscopic fundamental diagram based network traffic dynamics: a control systems perspective," Transportation Research Part B: Methodological, vol. 111, pp. 327-355, 2018.

[14] A. Selmoune, Q. Cheng, L. Wang, and Z. Liu, "Influencing factors in congestion pricing acceptability: a literature review," Journal of Advanced Transportation, vol. 2020, Article ID 4242964, 11 pages, 2020.

[15] M. S. Sheikh, J. Liang, and W. Wang, “An improved automatic traffic incident detection technique using a vehicle to infrastructure communication," Journal of Advanced Transportation, vol. 2020, Article ID 9139074, 14 pages, 2020.

[16] C. Ma, J. Zhou, X. Xu, and J. Xu, "Evolution regularity mining and gating control method of urban recurrent traffic congestion: a literature review," Journal of Advanced Transportation, vol. 2020, Article ID 5261580, 13 pages, 2020.

[17] Q. Hou, J. Leng, G. Ma, W. Liu, and Y. Cheng, "An adaptive hybrid model for short-term urban traffic flow prediction," Physica A: Statistical Mechanics and its Applications, vol. 527, p. $121065,2019$.

[18] M. Repoux, M. Kaspi, B. Boyac, and N. Geroliminis, "Dynamic prediction-based relocation policies in one-way station-based carsharing systems with complete journey reservations," Transportation Research Part B: Methodological, vol. 130, pp. 82-104, 2019.

[19] Q. Yang, Z. Shi, S. Yu, and J. Zhou, "Analytical evaluation of the use of leftturn phasing for single left-turn lane only," Transportation Research Part B: Methodological, vol. 111, pp. 266-303, 2018.

[20] L. L. Obsu, A. Meurer, S. M. Kassa, and A. Klar, "Modelling pedestrians'impact on the performance of a roundabout," Neural, Parallel, and Scientific Computations, vol. 24, pp. 317-334, 2016.

[21] X. Chen and M. S. Lee, "A case study on multi-lane roundabouts under congestion: comparing software capacity and delay estimates with field data," Journal of Traffic and Transportation Engineering (English Edition), vol. 3, no. 2, pp. 154-165, 2016.

[22] Y. Chitour and B. Piccoli, "Traffic circles and timing of traffic lights for cars flow," Discrete and Continuous Dynamical Systems Series B, vol. 5, no. 3, p. 599, 2005.

[23] S. K. Godunov, "A difference method for numerical calculation of discontinuous solutions of the equations of hydrodynamics," Matematicheskii Sbornik, vol. 89, no. 3, pp. 271-306, 1959.

[24] R. J. LeVeque, Finite Volume Methods for Hyperbolic Problems, volume 31, Cambridge University Press, 2002.

[25] S. N. Kruzhkov, "First order quasilinear equations in several independent variables," Matematicheskii Sbornik, vol. 123, no. 2, pp. 228-255, 1970. 